\title{
A CHECK-LIST OF THE MOSSES OF KAMCHATKA PENINSULA (FAR EAST)
}

\author{
IRINA V. CZERNYADJEVA ${ }^{1}$ \\ СПИСОК МХОВ ПОЛУОСТРОВА КАМЧАТКА (ДАЛЬНИЙ ВОСТОК)
}

\author{
ИРИНА В. ЧЕРНЯДЬЕВА ${ }^{1}$
}

Abstract

\begin{abstract}
Results of study of moss flora of Kamchatka Peninsula (Russian Far East) is presented. The list includes 439 species and 12 varieties of mosses. Annotations include localities and reference for each species. The history of bryological exploration and bibliography on bryophyte studies of Kamchatka Peninsula is given.
\end{abstract}

Резюме

Подведены итоги изучения флоры мхов полуострова Камчатка (Дальний Восток). В статье представлены история изучения бриофлоры полуострова и библиография по изучению его бриофлоры. Аннотированный список включает 439 видов и 12 разновидностей мхов. Для каждого вида перечислены все местонахождения на основе гербарных и литературных источников.

\section{HISTORY OF BRYOLOGICAL EXPLORATION}

The first collections of bryophytes from Kamchatka were made by the participants of the Krusenstern' round-the-world travel in 1803-1806. Bryophytes in this expedition were collected by W.G.Tilesius, while most of vascular plants - by G.H. Langsdorff; they collected in Kamchatka in the neighborhood of Avatcha Bay. Collections of Thilesius was determined and published by Wahlenberg (1811). This first publication on bryophytes of Kamchatka includes 31 mosses and 9 liverworts species. Based on the Kamchatka collection, Wahlenberg described two new species Encalypta corniculata (= Timiella corniculata) and Splachnum luteum var. melanocaulon (= Splachnum melanocaulon).

The next study in Kamchatka was conducted by A.L.C. Chamisso, who participated (together with J.F.G. von Eschscholtz and L. Choris) in the expedition of Kotzebue on «Rurik» in 1815-1818. This expedition explored surroundings of Petropavlovsk (Avacha Bay), where Chamisso gathered a small collection of mosses. This collection was studied by S.E. Bridel-Brideri, who included these data in his "Bryologia universa" (BridelBrideri, 1826-1827). In total, this paper includes
49 moss species. Also Bridel-Brideri reported 12 species for Kamchatka based on collections of Redowsky. However, as noted by H. Moeller (1927) and V.L. Komarov (1927), Redowsky died and did not reach Kamchatka, therefore his collections were most probably gathered near Ochotsk. Thus, the collections of Redowsky are not cited in the following checklist, and these data repeated by J.A. Weinmann (1845) and A.S. Lazarenko (1940, 1941a, 1941b, 1941-1945) are also omitted. Later Kamchatka was visited by the steamship «Blossom» during its around-the-world trip in 18251828, with the captain F.W. Beechey. In 1826 «Blossom» had a stop in Avatcha Bay, where the naval officer A. Collie and naturalist T. Lay gathered a small collection of plants, later studied and published by W.J. Hooker and G.A.W. Arnott (1841). Their list included 18 mosses and 1 liverwort. Later, publications of Erman (1835) and Wilson (1858) provided further data on mosses of Kamchatka, the latter publication included 10 moss species collected in Kamchatka by B. Seeman in the neighborhoods of Avatcha Bay. Thus, to the middle of XIX century, about 60 moss species were known from Kamchatka, mainly from the region of Avatcha Bay. A part of them were

1 - Россия 197376, Санкт-Петербург, ул. Проф. Попова, 2, Ботанический институт им. В. Л. Комарова РАН V. L. Komarov Botanical Institute Rus. Acad. Sci., Prof. Popov Str., 2, St. Petersburg, 197376 Russia 
mentioned by Weinman (1845), C. Mueller (18491851), S.O. Lindberg, H.W. Arnell (1890), V.F. Brotherus (1914, 1918, 1931).

In the beginning of XX century, two large overland expeditions considerably expanded existing knowledge of the nature of the Peninsula, including its bryoflora. In 1908-1909 F. P. Ryabushinsky organized the Kamchatka expedition, with the support of the Russian Geographic Society. The botanic exploration of the expedition was headed by V.L. Komarov, and his team included E.K. Bezais, L.G. Ramenski and V.P. Savicz. In 1908 they studied neighborhoods of Petropavlovsk, valley of Paratunka River, and also undetook a trip from eastern to western coast, up to the village Bolsheredsk. In 1909 the middle course of Kamchatka River and eastern coast were explored, and Bezais made a trip up to the mouth of Tigil River. The route of the expedition is described by Komarov (1927). The expedition resulted in a large herbarium, including numerous bryophyte specimens, collected mostly by Savicz and Ramenski, and to less degree by Komarov and Bezais. This collection was deposited in LE, and partly identified by L.I. Savicz. Using this collection she published "Flora of peat mosses of Kamchatka” (Savicz, 1932): 13 (of 27) species of Sphagnum were reported for Kamchatka for the first time. Families Funariaceae, Aulacomniaceae, Climaciaceae, Rhytidiaceae and Polytrichaceae were treated in the next publication (Savicz, 1934): it includes 28 species.

In 1920-1922 in the Swedish Kamtchatkan expedition studied the southern part of the peninsula from Petropavlovsk and Bolsheredsk up to Lopatka Cape. The botanist, E. Hulten and entomologist, R. Malaise participated in it. The routes of the expedition were summarized by Hulten (1927). Later, in 1924-1926, Malaise visited Kamchatka a few more times, exploring also central regions of the peninsula. Bryological collections of the expeditions are in S. Melin (1924) published identification of Sphagnum (14 species from Hulten collections from more than 30 localities) and Moeller (1927) - of other mosses (mostly collections of Hulten, some - of Malaise). Moeller (1.c.) included in his paper also data from earlier publications, thus providing the first extended account of mosses of Kamchatka that in- cluded 132 species and 12 subspecies. Almost a half of the species in that list were new for the peninsula. Later H. Persson studied the rest of Malaise collection and revised also specimens, identified by Moeller; his list includes 28 liverworts and 38 mosses (Persson, 1970).

In 1935-1936, the Complex Kamchatka Expedition of the Academy of Sciences of USSR explored the peninsula. Studies on geobotany and pedology were headed by L. N. Tjulina, who conducted the work on the western coast of Kamchatka, together with a geobotanist E. L. Ljubimova. Their bryophyte collections were determined by Lazarenko (and now they are in KW), who included them in his catalogue (Lazarenko 1940, 1941a, 1941b, 1941-1945). In this publication Lazarenko summarized all the available data on Far East of the USSR, indicating for Kamchatka 149 species.

In 1974-1978 Yu. N. Nechatayev collected bryophytes during his geobotanical studies in Kronozky Reserve. The results of that expedition were published (Rastitel'nost ..., 1994), inluding data on 60 moss species. Unfortunatelly the major part of the collection has been lost. The remaining part of this collection is stored in the Geobotany Dept. of Sankt-Petersburg State University; it was partly revised by the present author in 2005, revealing many misidentifications, so species from difficult groups nor re-studied by the present author are not included in the list.

In 1981 and 1990, M.S. Bocz studied peatlands in neighborhoods of Ossora, Avatcha Bay, Koryaky and Natchika. Her collections of Sphagnum were determined by E.O. Kuzmina, resulting in the publication of an annotated list of 27 species (Bocz, Kuzmina, 1991). Mosses collected by Bocz and also moss collection of O.A. Chernyagina were determined and published by G.V. Vyunova (1991). She listed 105 species of mosses. Unfortunately, collections of Bocz and part of this collection of Chrnyagina were lost. Remaining part of Chrnyagina collection is in herbarium of the Kamchatkan Branch of Pacific Insitute of Geography (Russian Academy of Sciences), with a few duplicates in LE. The available part of the Chernyagina collection was revised by the present author.

In 1979, V.Ya. Cherdantseva, a bryologist from 
VLAD, visited Kamtchatka and then published a list of 106 mosses (based on collections of S.V. Osipov and herself, the latter collected in 21 localities, mainly along the road Petropavlovsk Ust'-Kamtchatsk - Esso, and also around Avatcha Bay (Cherdantseva, Osipov, 1998). Scattered collections from Kamchatka were published by Cherdantseva, also in various papers (Cherdantseva, 1978, 1989, 1993), including list of 78 species from Kronozky Reserve (Cherdantseva, 2003).

In 1990 and 2001-2005 the present the author carried out the bryophyte studies in different parts of the Peninsula. In the framework of the full scale exploration of South Kamchatskiy Reserve, the basins of Left and Right Kihchik, Nachilova, Bannaya and Elovka Rivers, vicinity of Ossora, Esso, Pushchino, Kljuchi, Kosyrevsk, Krutoberegovo settelmens, vicinity of Shiveluch and Mutnovskj volcanoes, vicinity of Shadutka Bay, on Kluchevskaya group of volcanoes: on western slope of Ushkovsky volcano on "Kljuchevskoj Dol”, vicinity of Kopyto Mt. and Bilchenok glacier. Moreover, the collections of O.A. Chernyagina from Kirevna River, volcano Mutnovskij, the Pushchino hot springs and the small collection of V. Bakalin from Middle Range were determined by the author. The resalts of investigations of the separate local floras, as well as history of the bryoflora of Kamchatka were published (Czernyadjeva, 1995a; 2002a; 2002b; 2002c; 2003b; 2005b; Czernyadjeva \& Potemkin, 2002; 2003; Czernyadjeva \& al., 2005; Czernyadjeva \& Afonina, 2005).

The complex investigations of plant communities were carried out by geobotanists in cooperation with bryologists and lichenologists. Information concerning moss cover of these communities were published by Neshatayeva et al. (1997, 2003, 2004, 2005, 2006).

New and interesting discoveries of mosses from Kamchatka were published in a series of papers: Bednarek-Ochyra, Czernyadjeva (2003); Czernyadjeva (1995b,c; 2000; 2003a; 2004; 2005a; Czernyadjeva, Ignatova, 2004). All author' collection are stored at Komarov Botanic Institute (LE).

\section{REGIONS OF KAMCHATKA}

The accepted regions of Kamchatka (Fig. 1) are mostly physiographic and based on subdivision of Kamchatka by Kharkevich (1981) and
Yakubov \& Chernyagina (2004), with some amendations:

Northern Kamchatka includes the territory north from the line Palana settlement - the northern point of Osernyii Peninsula. Forest vegetation is confined to river valleys, Pinus pumila and Alnus fruticosa shrubs dominate.

Western Kamchatka includes the territory of Western Kamchatka Lowland, characterizied by numerous bogs alternating with Betula ermanii forest.

Middle Kamchatka includes mountains of the Middle Range, with elevations ca. (300-)4001200(-3621) m.

Central Kamchatka includes the most part of the valley of Kamchatka River, characterized by forests of Betula platyphylla, Larix cajanderi, Picea ajanensis and in flood plain - forests of trees of Salicaceae.

Eastern Kamchatka includes the Eastern Range, with main volcanos of the Peninsula, (300-)800-1700 (-4750) m.

Southern Kamchatka is a mountain region south from the line Petropavlovsk City - Ust'Bolscheredsk City. The main part of border is the Plotnikova River, which is an important limit for many species of vascular plants.

\section{LIST OF SPECIES}

The check-list includes all the confirmed herbarium specimens, as well as literature records which are considered to be reliable by various reasons. The species that are included based on only literature data are marked by $(*)$. The dubious records are marked (?). Localities are given in bold (cf. Fig. 1) and they are followed by a number denoting the sourse of information given in the section "Sources of information..." before Literature Cited section.

Abietinella abietina (Hedw.) M. Fleisch. - N1: 10, W10: 14, M15: 33, 36, M17: 8, 36, C18: 20, C19: 20, E27: 36, E29: 36, E31: 36, E34.: 30, 9, S54: 15, S55: 15 .

Amblystegium serpens (Hedw.) B.S.G. - W9: 28, W10: 14,; W11: 16, M15: 33, 36, M17: 8, C18: 20, C25: 21, E27: 36, E28: 36, E29: 36, E31: 36, S41: 16, S55: 15 .

Amphidium lapponicum (Hedw.) Schimp. - W10: 14, M17: 8, C18: 20, E27: 36, E29: 36, E32: 8, S41: 16, S51: 36 . 
Fig. 1. Regions of Kamchatka and collecting localities (in text used with the first letter of region: W5 - from Western Kamchatka, S42 - from Southern, etc.).

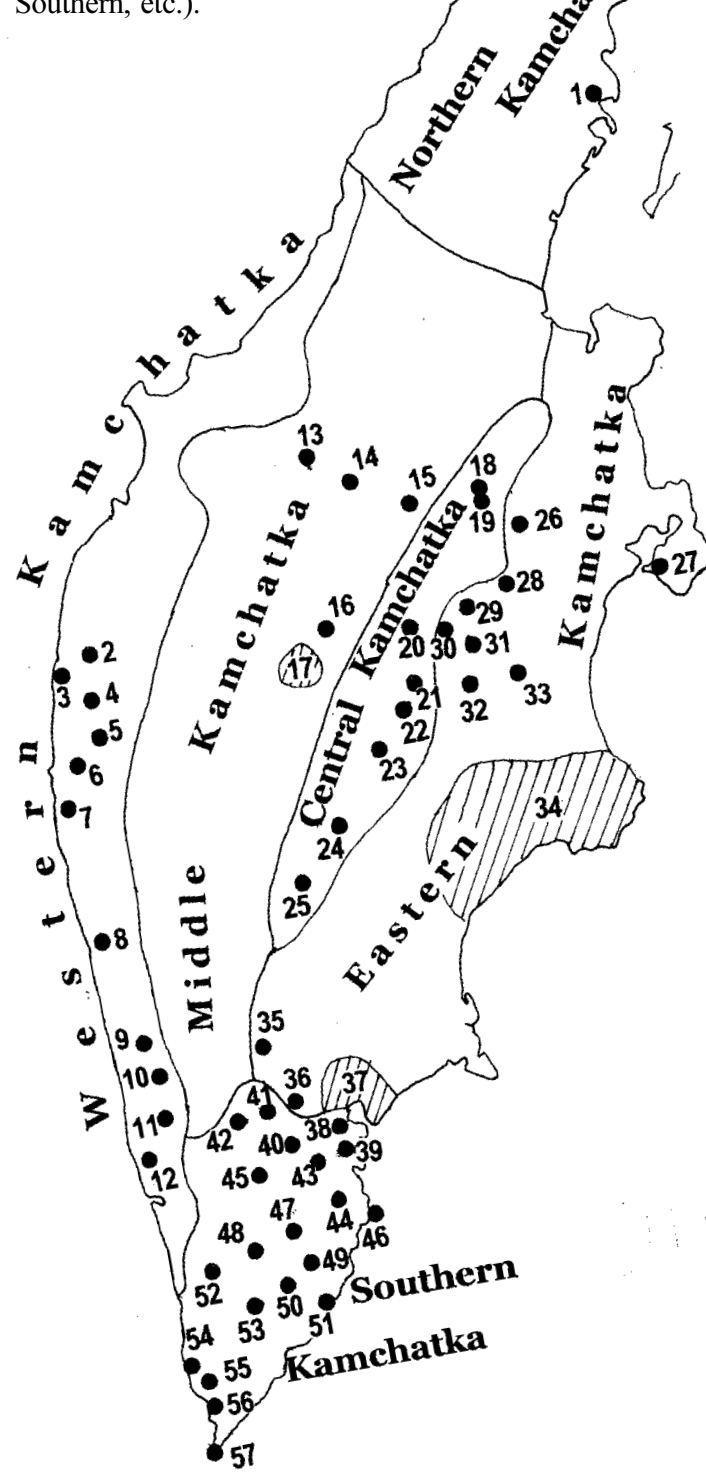

NORTHERN KAMCHATKA

N1 - Vicinity of Ossora Settl., incuding Kaspa Mt. and Karaginskij Is., $\sim 59^{\circ} 16^{\prime} \mathrm{N}, 163^{\circ} 01^{\prime} \mathrm{E}$, alt. 10-400 $\mathrm{m}$ - окрестности пос. Оссора, включая гору Каспа и о. Карагинский (Cherdantseva, 1978; Vyunova, 1991; Czernyadjeva, 1995).

\section{WESTERN KAMCHATKA}

W2 - Sopochnaya River, incuding Moroshechnaya River, and Soichek River, $\sim 56^{\circ} 04^{\prime} \mathrm{N}, 155^{\circ} 55^{\prime} \mathrm{E}$, alt. 10-200 m - p. Сопочная, включая pp. Морошечная и Соичек (Lazarenko, 1940, 1941b).
W3 - Icha River, incuding Icha village and Icha fishy factory, $\sim 55^{\circ} 35^{\prime} \mathrm{N}, 155^{\circ} 57^{\prime} \mathrm{E}$, alt. $10-200 \mathrm{~m}-\mathrm{p}$. Ича, включая бывший пос. Ича и Ичинский рыбкомбинат (Lazarenko, 1940, 1941a, 1941b, 1945).

W4 - Oblukovina River, $\sim 55^{\circ} 17^{\prime} \mathrm{N}, 155^{\circ} 39^{\prime} \mathrm{E}$, alt. 10-200 m - p. Облуковина (Lazarenko, 1940, 1941a, 1945).

W5 - Krutogorova River, including Koopa River and Polovinka River, $\sim 55^{\circ} 03^{\prime} \mathrm{N}, 155^{\circ} 40^{\prime}$ E, alt. $10-200$ $\mathrm{m}$ - p. Крутогорова, включая p. Копа и р. Половинка (Savicz, 1932; Lazarenko, 1940, 1941a, 1941b, 1945; Cherdantseva, 1993).

W6 - The interfluve of Krutogorova and Kolpakova Rivers, $\sim 54^{\circ} 51^{\prime} \mathrm{N}, 155^{\circ} 43^{\prime} \mathrm{E}$, alt. 10-200 m междуречье pр. Крутогорова и Колпакова ( Vyunova, 1991).

W7 - Kolpakova River, including Kunzhik River, $\sim 54^{\circ} 42^{\prime} \mathrm{N}, 155^{\circ} 46^{\prime}$ E, alt. 10-200 m - p. Колпакова, включая р. Кунжик (Lazarenko, 1940, 1941a, 1941-45).

W8 - Sobolevo Settl., including Vorovskaya River, Vorovskoj fishy factory, $\sim 54^{\circ} 19^{\prime} \mathrm{N}, 156^{\circ} 09^{\prime} \mathrm{E}$, alt. 10-200 m - пос. Соболево, включая р. Воровская и рыбкомбинат Воровской (Savicz, 1932; Lazarenko, 1940, 1941a, 1941b, 1945).

W9 - Right Kihchik River, including Kihchik village and Kol' River, $\sim 53^{\circ} 35^{\prime}$ N, $156^{\circ} 41^{\prime}$ E, alt. $\sim 200 \mathrm{~m}$ - бассейн р. Правый Кихчик, включая с. Кихчик, p. Коль (Savicz, 1932; Lazarenko, 1940, 1941a, 1941b; Neshataeva \& al., 2005; author data).

W10 - Left Kihchik River, $53^{\circ} 25^{\prime}$ N, $156^{\circ} 40^{\prime}$ E, alt. 200-500 m - бассейн р. Левый Кихчик (Czernyadjeva, 2002).

W11 - The interfluve of lower Bolshaya-Bystraya River and middle Nachilova River, 53 ${ }^{\circ} 06^{\prime} \mathrm{N}, 156^{\circ} 53^{\prime}$ E, alt. 250-600 m - междуречье нижн. теч. p. Быстрой-Большой и ср. теч. p. Начилова (Czernyadjeva, Potemkin, 2003).

W12 - Ust'-Bolscheredsk Sity, including lower Bolshaya-Bystraya River, Zykovaja village, $\sim 52^{\circ} 49^{\prime} \mathrm{N}$, $156^{\circ} 12^{\prime}$ E, alt. 10-50m, - г. Усть-Большерецк, включая низовья р. Большой и дер. Зыкова (Melin, 1924; Moeller, 1927; Savicz, 1932; Cherdantseva, Osipov, 1998).

\section{MIDDLE KAMCHATKA}

M13 - Middle Range, Tigil' River, nearly Belaya River, $\sim 56^{\circ} 39^{\prime} \mathrm{N}, 159^{\circ} 01^{\prime} \mathrm{E}$, alt. $450 \mathrm{~m}$ - Срединный хр., p. Тигиль в районе впадения р. Белая (Coll. V.A. Bakalin, det. I.V. Czernyadjeva).

M14 - Middle Range, western slope of Alnej vol., valley of Shirokij glacier, $\sim 56^{\circ} 39^{\prime} \mathrm{N}, 159^{\circ} 29^{\prime} \mathrm{E}$, alt. 1000-1600 m - Срединный хр., зап. склон влк. Алней, долина ледника Широкий (Coll. V.A. Bakalin, det. I.V. Czernyadjeva).

M15 - Middle Range, Alnej vol., valley of Kirev- 
na River, Verhnekireunsky hot springs, $56^{\circ} 45^{\prime} \mathrm{N}$, $160^{\circ} 00^{\prime}$ E, alt. $500 \mathrm{~m}$ - Срединный хребет, влк. Алней, долина р. Киревна, Верхнекиреунские горячие источники (Vyunova, 1991; Coll. O.A. Chernyagina, det. I.V. Czernyadjeva).

M16 - Middle Range, vicinity of Anauna River Срединный хребет, окрестности р. Анаун (Moeller, 1927; Persson, 1970).

M17 - Middle Range, vicinity of Esso Settl., $\sim 55^{\circ} 56^{\prime} \mathrm{N}, 158^{\circ} 41^{\prime} \mathrm{E}$, alt. 300-1600 m - Срединный хребет, окрестности пос. Эссо (Cherdantseva, 1993; Cherdantseva, Osipov, 1998; author data).

\section{CENTRAL KAMCHATKA}

C18 - Upper Elovka River, 56 $55^{\prime} \mathrm{N}, 160^{\circ} 59^{\prime} \mathrm{E}$, alt. 150м - верхнее теч. p. Еловка (Czernyadjeva, 2005b).

C19 - Middle Elovka River, 56 $53^{\circ}$ N, 160 $55^{\circ}$ E, alt. 150м - среднее теч. p. Еловка (Czernyadjeva, 2005b).

C20 - Vicinity of Kosyrevsk Settl., $\sim 56^{\circ} 03^{\prime} \mathrm{N}$, $159^{\circ} 54^{\prime}$ E, alt. 50-150м - окрестности пос. Козыревск (Moeller, 1927; Savicz, 1934; Persson, 1970; Cherdantseva, 1993; Cherdantseva, Osipov, 1998; author data).

C21 - Vicinity of Tolbatchick village, $\sim 55^{\circ} 33^{\prime} \mathrm{N}$, $159^{\circ} 57^{\prime}$ E - окрестности с. Толбачик (Savicz, 1934; Moeller, 1927; Persson, 1970).

C22 - Vicinity of Shtchapina village, including Shtchapinskj hot springs and Nikolka Mt. , $\sim 55^{\circ} 21^{\prime} \mathrm{N}, 159^{\circ} 26^{\prime}$ Е - окрестности с. Щапино, включая Щапинские горячие источники и окрестности г. Николка (Savicz, 1932, 1934; Moeller, 1927; Persson, 1970).

C23 - Vicinity of Kirgannik village, including of village Mashura, $54^{\circ} 49^{\prime} \mathrm{N}, 158^{\circ} 47^{\prime} \mathrm{E}$ - окрестности с. Кирганник, включая с. Машура (Savicz, 1932, 1934; Moeller, 1927).

C24 - Vicinity of Sharomy village, $\sim 54^{\circ} 24^{\prime} \mathrm{N}$, $158^{\circ} 11^{\prime}$ E - окрестности с. Шаромы (Savicz, 1934).

C25 - Vicinity of Pushchino Settl., including Pushchincky hot springs, $\sim 54^{\circ} 11^{\prime} \mathrm{N}, 158^{\circ} 01^{\prime} \mathrm{E}$, alt. 300 $600 \mathrm{~m}$ - окрестности пос. Пущино, включая Пущинские горячие источники (Savicz, 1934; Czernyadjeva, Afonina, 2005).

\section{EASTRERN KAMCHATKA}

E26 - Vicinity of Shiveluch vol., $\sim 56^{\circ} 33^{\prime} \mathrm{N}, 161^{\circ} 15^{\prime}$ E, alt. $200-800 \mathrm{~m}$, including forest nursery, $56^{\circ} 27^{\prime} \mathrm{N}$, $161^{\circ} 01^{\prime}$ Е - окрестности вул. Шивелуч, включая лесхоз (Cherdantseva, Osipov, 1998; author data).

E27 - Kamchatskij Peninsula, vicinity of Krutoberegovo Settl., $\sim 56^{\circ} 13^{\prime} \mathrm{N}, 162^{\circ} 50^{\prime} \mathrm{E}$, alt. 10-400 m п-ов Камчатский, окрестности пос. Крутоберегово (author data).

E28 - Vicinity of Kljuchi Settl., $\sim 56^{\circ} 20^{\prime} \mathrm{N}, 160^{\circ} 50^{\prime}$ E, alt. 10-100 m - окрестности пос. Ключи (Cherdantseva, Osipov, 1998; author data).

E29 - Kluchevskaya group of volcanos, vicinity of Bilchenok glacier, $\sim 56^{\circ} 11^{\prime} \mathrm{N}, 160^{\circ} 21^{\prime} \mathrm{E}$, alt. 600$1500 \mathrm{~m}$ - Ключевская группа вулканов, окрестности ледника Бильченок (author data).

E30 - Kluchevskaya group of vol., vicinity of Kopyto Mt., $55^{\circ} 57^{\prime} \mathrm{N}, 160^{\circ} 08^{\prime} \mathrm{E}$, alt. $350-700 \mathrm{~m}-$ Ключевская группа вулканов, окрестности г. Копыто (author data).

E31 - Kluchevskaya group of vol., western slope of Ushkovsky vol., upper Shirokij stream, "Kljuchevskoj Dol”, $\sim 55^{\circ} 58^{\prime} \mathrm{N}, 160^{\circ} 17^{\prime} \mathrm{E}$, alt. $900-1600 \mathrm{~m}-$ Ключевская группа вулканов, Зап. склон вул. Ушковский, верховья руч. Широкий, «Ключевской дол» (author data).

E32 - Kluchevskaya group of vol., Tolbatchick vol., $55^{\circ} 46^{\prime} \mathrm{N}, 160^{\circ} 15^{\prime} \mathrm{E}$, alt. $\sim 1000 \mathrm{~m}-$ Ключевская группа вулканов, вул. Толбачик (Persson, 1970; Cherdantseva, Osipov, 1998).

E33 - Kluchevskaya group of vol., middle Bolshaya Hapica River, $55^{\circ} 56^{\prime} \mathrm{N}, 161^{\circ} 12^{\prime}$ E, alt. $\sim 100$ $\mathrm{m}$ - Ключевская группа вулканов, среднее теч. $\mathrm{p}$. Большая Хапица (author data).

E34 - Kronotsky Reserve - Кроноцкий заповедник (Savicz, 1932, 1934; Rastitel' nost...1994; Cherdantseva, 1989, 1993, 2003; Coll. of expedition of Yu.N. Neshataev, det. I.V. Czernyadjeva).

E35 - Vicinity village Malki, including Poperechnaya Mt., Poperechnaya River and Ganalskaya tundra, $\sim 53^{\circ} 20^{\prime} \mathrm{N}, 157^{\circ} 26^{\prime} \mathrm{E}$ - окрестности с. Малки, включая г. Поперечная, р. Поперечная и Ганальскую тундру (Savicz, 1932, 1934; Cherdantseva, 1989; Cherdantseva, 1993).

E36 - Vicinity Settl. Natchiki, $\sim 53^{\circ} 06^{\prime} \mathrm{N}, 157^{\circ} 44^{\prime}$ Е - окрестности с. Начики (Melin, 1924; Moeller, 1927; Savicz, 1932, 1934; Vyunova, 1991).

E37 - Vicinity Petropavlovsk Sity, including Elizovo Sity (Savoiko), Korjakski and Avatcha vol., Korjaka Settl., Korjakskaya River, north and west coasts Avatcha Bay, Nalychevskoe Lake, $\sim 53^{\circ} 05^{\prime} \mathrm{N}, 158^{\circ} 41^{\prime} \mathrm{E}$, alt. 10$400 \mathrm{~m}$ - окрестности г. Петропавловска-Камчатского, включая г. Елизово (Завойко), вул. Коряцкий и Авача, пос. Коряки, р. Корякская, северное и западное побережья Авачинской бухты, оз. Налычевское (Wahlenberg, 1811; Bridel-Brideli, 1826-1827, Hooker, Arnott, 1841; Wilson, 1858; Melin, 1924; Moeller, 1927; Savicz, 1932, 1934; Persson, 1970; Vyunova, 1991; Cherdantseva, Osipov, 1998; Czernyadjeva, 2000).

\section{SOUTHERN KAMCHATKA}

S38 - Vicinity Settl. Paratunka, including Settl. Termal' nyi and Nikolaevsk, $\sim 52^{\circ} 44^{\prime} \mathrm{N}, 158^{\circ} 11^{\prime} \mathrm{E}$, alt. 15 $150 \mathrm{M}$ - окрестности пос. Паратунька, включая пос. Термальный и Николаевск (Moeller, 1927; Savicz, 1932; Vyunova, 1991; Cherdantseva, Osipov, 1998; author data).

S39 - Upper Viljucha River and Settl. Rodnikovyi, 
$52^{\circ} 39^{\prime} \mathrm{N}, 158^{\circ} 15^{\prime} \mathrm{E}$, alt. 300м - верховья р. Вилюча и пос. Родниковый (Cherdantseva, Osipov, 1998).

S40 - Kohelok Mt., including Kaach Mt., - г. Кохелок, включая г. Каач (Moeller, 1927).

S41 - Middle Bannaja River, including hot springs, $52^{\circ} 54^{\prime} \mathrm{N}, 157^{\circ} 30^{\prime} \mathrm{E}$, alt. 200-600 m - среднее течение p. Банная, включая горячие источники (Moeller, 1927; Czernyadjeva, Potemkin, 2003).

S42 - Basin Karymchina River, including Agash$\mathrm{ka}$, Siku, Ipelka Rivers, $\sim 52^{\circ} 52^{\prime} \mathrm{N}, 157^{\circ} 07^{\prime} \mathrm{E}$ - бассейн p. Карымчина, включая pp. Агашка, Сику, Ипелька (Melin, 1924; Moeller, 1927).

S43 - Gorelji vol., $52^{\circ} 35^{\prime} \mathrm{N}, 158^{\circ} 00^{\prime}$ E, alt. $\sim 1100$ m - вул. Горелый (Moeller, 1927; Cherdantseva, Osipov, 1998).

S44 - Skalistaya Mt., slope Mutnovskyi vol., 52³2' $\mathrm{N}, 158^{\circ} 12^{\prime} \mathrm{E}$, alt. 800-1000 m - г. Скалистая на склоне вул. Мутновский (Cherdantseva, Osipov, 1998; Czernyadjeva et all., 2005).

S45 - Opala vol., including middle Opala River, $\sim 52^{\circ} 33^{\prime} \mathrm{N}, 157^{\circ} 19^{\prime} \mathrm{E}$ - вул. Опала, включая среднее течение р. Опала (Melin, 1924; Moeller, 1927; Persson, 1970).

S46 - Vicinity Russkaya (Akhomten) Bay, $\sim 52^{\circ} 25^{\prime}$ $\mathrm{N}, 158^{\circ} 25^{\prime} \mathrm{E}$ - окрестности бух. Русская (Ахомтен) (Melin, 1924; Moeller, 1927; Persson, 1970).

S47 - Assatcha Mt. , $\sim 52^{\circ} 21^{\prime} \mathrm{N}, 157^{\circ} 50^{\prime} \mathrm{E}-$ r. Асача (Melin, 1924; Moeller, 1927).

S48 - Upper Savan River - верхнее течение p. Саван (Moeller, 1927).

Anacamptodon kamchaticum Czernyadjeva - W10: 17, E27: 36,

Andreaea alpestris (Thed.) Sharp-C18: 20, S44: 22. - nivalis Hook. - S43: 8, S44: 22, S46: 27, S55: 15.

- rupestris Hedw. var. papillosa (Lindb.) Podp. - N1: 10, M14 : 36, E29: 36, S55: 15.

- rupestris Hedw. var. rupestris - W10: 14, M17: 8, C18: 20, E27: 36, E29: 36, S41: 16, S55: 15.

Anomobryum julaceum (P. Gaertn. et al.) Schimp. var. concinnatum (Spruce) Lindb. - E29: 36, E31: 36.

Anomodon longifolius (Brid.) C. C. Hartm. - W9: 28.

- viticulosus (Hedw.) Hook. et Taylor - W10: 14. Aongstroemia longipes (Sommerf.) B. S. G. - E33: 36. Arctoa fulvella (Dicks.) B.S.G. - M17: 8, E29: 36, S41: 16, 27, S44: 8, 22, S55: 15.

Atrichum tenellum (Roell) B.S.G. - W11: 16. Aulacomnium palustre (Hedw.) Schwaegr. var. palustre-N1: 10, 33, W10: 14,; W11: 16, W12: 27, W6: 33, W9: 36, M14 : 36, M15: 33, 36, M17: 8, 36, C18: 20, C20: 36, C21: 32, C22: 32, C22: 32, C23: 32, C24: 32, C25: 21, E26: 36, E27: 36, E28: 36 , E29: 36, E30: 36, E31: 36, E34:: 9, 30, 32, E35: 32, E36: 32, E37: 23,27, 32, S41: 16,27, S51: 36,
S49 - Shadutka vol., including upper Shadutka River, $\sim 52^{\circ} 04^{\prime} \mathrm{N}, 157^{\circ} 43^{\prime} \mathrm{E}$ - вул. Ходутка, включая верхнее течение р. Ходутка (Moeller, 1927; Persson, 1970).

S50 - Ksudach vol. , 51 ${ }^{\circ} 49^{\prime} \mathrm{N}, 157^{\circ} 40^{\prime} \mathrm{E}$ - вул. Ксудач (Moeller, 1927).

S51 - Vicinity Shadutka Bay, $51^{\circ} 46^{\prime} \mathrm{N}, 158^{\circ} 22^{\prime} \mathrm{E}$ , alt. 5-30 m- окрестности бух. Ходутка (author data).

S52 - Vicinity Golygino Settl., including lower Opala River - окрестности бывшего пос. Голыгино, включая низовья р. Опала (Moeller, 1927).

S53 - Sahach vol., including upper Unkanakchek River - вул. Сахач, включая верхнее течение p. Унканакчек (Melin, 1924; Moeller, 1927; Persson, 1970).

S54 - Vicinity Settl.Ozernoi, including lower Ozernaj River and lower Javina River, $\sim 51^{\circ} 30^{\prime} \mathrm{N}, 156^{\circ} 30^{\prime}$ E, alt. $20 \mathrm{~m}$ - окрестности пос. Озерной, включая низовья р. Озерная и низовья р. Явинской (Moeller, 1927; Persson, 1970; Czernyadjeva, Potemkin, 2002).

S55 - Koshelevskyi vol., including vicinity Paushchetka Settl., lower Vtoraya, Tret'ya, Chetvertaya, Holodnaya Rivers, $\sim 51^{\circ} 31^{\prime} \mathrm{N}, 156^{\circ} 39^{\prime} \mathrm{E}$, alt. $10-1000 \mathrm{~m}$ - вул. Кошелевский, включая окр. пос. Паужетка, низовья рр. Вторая, Третья, Четвертая, Холодная (Czernyadjeva, Potemkin, 2002; data of E. Ignatova).

S56 - Lower Kambolnaya River, including Toporkoff Island, $\sim 51^{\circ} 10^{\prime} \mathrm{N}, 156^{\circ} 43^{\prime} \mathrm{E}$ alt. $\sim 30 \mathrm{M}-$ низовья p. Камбальная, включая о. Топорков ( Melin, 1924; Moeller, 1927; Czernyadjeva, Potemkin, 2002).

S57 - Cape Lopatka, $\sim 50^{\circ} 54^{\prime} \mathrm{N}, 156^{\circ} 39^{\prime} \mathrm{E}$ alt. $\sim 5 \mathrm{~m}$ - мыс Лопатка (Moeller, 1927).

S53: 27, S55: 15, S56: 15.

- palustre (Hedw.) Schwaegr. var. imbricatum B. S. G. - E26: 36.

- turgidum (Wahlenb.) Schwaegr. - N1: 10, W10: 14, M15: 36, M17: 8, 36, E26: 36, E29: 36, E31: 36, E34.: 32, E37: 32, E36: 32, S43: 8, 27, S45: 27, S55: 15 , S56: 15 .

Barbula cf. convoluta Hedw. - M15: 36,

— unguiculata Hedw. - W10: 14.

Bartramia ithyphylla Brid. - N1: 10, W10: 14, M15: 33, M17: 8, 36, C25: 21, E27: 36, E29: 36, E31: 36, E37: 4, S51: 36, S55: 15.

- pomiformis Hedw. - W10: 14, M15: 33, 36, M17: 8, C18: 20, C20: 8, C25: 21, E27: 36, E29: 36, E37: 4, 8, S38: 8, S41: 16, S51: 36 .

Bartramiopsis lescurii (James) Kindb. - W5: 7, W10: 14, E34: 7, E35: 32, E37: 32, S39: 8.

Brachytheciastrum collinum (Schleich. ex Muell. Hal.) Ignatov et Huttunen - E29: 36.

_ *velutinum (Hedw.) Ignatov et Huttunen - E37: 27.

Brachythecium albicans (Hedw.) B.S.G. - W9: 28, M17: 8, C25: 21, E29: 36, E31: 36.

- buchananii (Hook.) A. Jaeger - C18: 20.

—*? campestre (Muell. Hal.) B.S.G. - W5: 25. 
- cirrosum (Schwaegr.) Schimp. - E31: 36.

— erythrorrhizon B.S.G. s.1. - W9: 28, W10: 14, M15: 36, C18: 20, C19: 20, C25: 21, E29: 36, S41: 16.

- mildeanum (Schimp.) Schimp. ex Milde -W8: 25, W9: 28, M15: 36, C18: 20, C25: 21, E34: 36, S41: 16, S55: 15, S56: 15.

— rivulare B.S.G. - W8: 25, W9: 28, W10: 14, W11: 16, M15: 33, 36, C18: 20, C25: 21, E27: 36, E28: 36, E29: 36, E33: 36, E34.: 9, S38: 27, S41: 16, S42: 27 , S55: 15 .

— rotaeanum De Not. - W9: 28, W10: 14, W11: 16, C25: 21, S41: 16.

- salebrosum (F. Weber et D. Mohr) Schimp. - N1: 10, W4: 25, W5: 25, W8: 25, W9: 28, W10: 14, W11: 15, M15: 33, 36, C18: 20, C19: 20, C25: 21, E28: 36, E29: 36, E33: 36, E34. 30, E37: 27, S41: 16, S42: 27, S55: 15, S56: 15.

— turgidum (C. C. Hartm.) Kindb. - E29: 36, E31: 36, S54: 15, S55: 15.

— udum (J. I. Hagen) J. I. Hagen - E29: 36.

Breidleria pratensis (L. F. Koch ex Spruce) Loeske N1: 10, W11: 16, S41: 16, S55: 15, Error: 30.

Bryhnia hultenii Bartr. - W9: 28, W10: 14, W11: 16, E37: 29, S41: 16, S51: 36, S55: 36.

- novae-angliae (Sull. et Lesq.) Grout. -W2: 25, W4: 25, W9: 25, 28, W10: 14, C25: 21, E37: 29, S49: 29, S54: 15, S55: 15. Many specimens of this species don't have spine in end of nerve and can be classified as intermediate between $B$. novae-angli$a e$ and $B$. hultenii. More detailed studies are essencial for specimens of Bryhnia from Far East.

Bryoerythrophyllum recurvirostrum (Hedw.) P.C. Chen -W9: 28, W10: 14, M17: 8, C18: 20, C20: 8, C25: 21, E28: 36,E29: 36, E31: 36, E34: 9, E37: 4, 8, S55: 15 .

Bryoxiphium norvegicum (Brid.) Mitt. var. japonicum (Berggr.) A. et D. Loeve - W5: 25, M17: 36, C20: 8, E27: 36, E29: 36, E30: 36, E34: 9, E36: 37, S41: 16. The length of excurrent costa of perichaetial leaves differs significantly even within a specimen, so it is difficult to classify many specimens between var. norvegicum and var. japonicum.

Bryum alpinum J. Huds. ex With. - S44: 22.

—amblyodon Muell. Hal. - W10: 14, E29: 36, E37: 35, S41: 16, S54: 27, S55: 15.

— archangelicum B.S.G. - W10: 14, E29: 36.

- argenteum Hedw. - W10: 14, W11: 16, M15: 33, C18: 20, C20: 36, E29: 36, E31: 36, E34: 9, E37: 4, S38: 36, S41: 16.

- bimum (Schreb.) Turner - C25: 21,

- caespiticium Hedw. - C25: 21, E29: 36, E37: 23.

- capillare Hedw. - W9: 36, E29: 36, E32:8, E37: 34, 29, S41: 16.

— creberrimum Taylor-M15: 36, C25: 21, E29: 36 , S41: 16 .
- cryophilum O. Maort-C25: 21.

- cyclophyllum s.l. (Schwaegr.) B.S.G. - M17: 36, C25: 21, S41: 16, S55: 15.

- elegans Nees ex Brid. - M15: 36, S44: 22.

- intermedium (Brid.) Blandow - C25: 21, E37: 35, S44: 22.

— laevifilum Syed-W11: 16, C18: 20, C25: 21, S41: 16, S44: 22.

- lonchocaulon C. Muell. - W9: 36, E29: 36,

- pallens Sw. - C25: 21.

_ * pallescens Scheich. ex Schaegr. - E32: 8, E37: 4.

- pseudotriquetrum (Hedw.) P. Gaertn. et al. - N1: 10, W9: 28, W10: 14, W11: 16, M15: 33, 36, M17: 36, C25: 21, S41: 16, S44: 22, S55: 15.

- schleicheri Schwaegr--W10: 14, M16: 29, M17: 36 , C25: 21, E27: 36, E29: 36, E34: 9, E37: 35, 29, S41: 16, S42: 27, S44: 22, S55: 15 . Some authors pointed B. schleicheri var. latifolium (Schwaegr.) Schimp for the Kamchatka region. Author according to Zolotov, 2000 , includes var. latifolium in the typical variety.

— *subneodamense Kindb. - W12: 27,

— *uliginosum (Brid.) B.S.G. -W12: 27.

— weigelii Spreng. in Bischler - W10: 14, M14 : 36, C25: 21, E27: 36, E34: 9, S44: 22, S55: 15.

Bucklandiella microcarpa (Hedw.) Bednarek-Ochyra et Ochyra-C25: 21, E29 36, E34: 9, S41: 16, Error 33 .

- nitidula (Cardot) Bednarek-Ochyra et Ochyra W10: 36.

- sudetica (Funck) Bednarek-Ochyra et Ochyra-N1: 10, W10: 14, M15: 36, E29 36, S41: 16, S44: 22, S55: 15 .

- sudetica f. kindbergii (Frisvoll) Bednarek-Ochyra et Ochyra - S44: 22, S55: 15.

Buxbaumia aphylla Hedw. - M17: 7, 36, S41: 16.

Callicladium haldanianum (Grev.) H.A. Crum - W9: 28, W10: 14, W11: 16, S41: 16.

Calliergon cordifolium (Hedw.) Kindb. - N1: 33, 10, W3: 25, W9: 28, W10: 14, W11: 16, W12: 27, M17: 36, C18: 20, C25: 21, E34: 9, 36, E37: 27, S38: 36, S41: 16, S48: 27, S50: 27, S51: 36, S55: 15.

— giganteum (Schimp.) Kindb. - W9: 36, E37: 27, 33, S46: 27.

— megalophyllum Mikut. - S51: 36.

— richardsonii (Mitt.) Warnst. - W10: 14, S56: 15.

Calliergonella cuspidata (Hedw.) Loeske - M15: 36, E34: 36, E36: 37, E37: 23, S56: 15.

- lindbergii (Mitt.) Hedenaes - W10: 14, M14: 36, C19: 20, C20: 36, C25: 21, E29: 36, S38: 36, S41: 16, S50: 27, S55: 15.

Campyliadelphus chrysophyllus (Brid.) Kanda-M15: 33, C25: 21, E29: 36, E31: 36, S41: 16, S55: 15.

Campylidium hispidulum (Brid.) Ochyra - W9: 28, W10: 14, W11: 16, C18: 20, C25: 21, E28: 36, E29: 36, S41: 16 . 
- sommerfeltii (Myr.) Ochyra-N1: 10, W9: 28, C18: 20, C25: 21, E34: 9, 30, S41: 16, S55: 15. The differences of $C$. sommerfeltii and C. hispidulum in material from Kamchatka are rather small, so it is anticipated, that after a revision they may be combined.

Campylium protensum (Brid.) Kindb. - C25: 21.

- stellatum (Hedw.) C. E. O. Jensen-N1: 10, E27: 36 , E29: 36, E31: 36, E37: 27, 33, S51: 36, S56: 15.

Campylopus atrovirens De Not. - S55: 38 .

— subulatus Schimp. - E31: 36, S38: 38, S55: 38.

- schimperi Milde - W10: 14

Ceratodon heterophyllus Kindb. - C25: 21, S44: 22.

- purpureus (Hedw.) Brid. - N1: 10, W9: 28, W10: 14, W11: 16, W12: 27, M13: 36, M15: 33, 36, M17: 8, 36, C18: 20, C19: 20, C20: 8, 36, C25: 21, E26: 8, 36, E27: 36, E28: 36, E29: 36, E30: 36, E31: 36, E32: 8, E33: 36, E34: 9, E37: 27, 34, 35, S38: 36, S40: 27, S41: 16 , S43: 27, S44: 8 , 22, S46: 27, S51: 36, S54: 15 , S55: 15 , S56: 15 .

Cinclidium latifolium Lindb. - S56: 15.

- stygium Sw. - Warnstorf 1911-17: without location; E31: 36, S56: 15.

- subrotundum Lindb. - E34. - 36, S51: 36.

Claopodium pellucinerve (Mitt.) Besch. - W10: 14, S51: 36 .

Climacium dendroides (Hedw.) F. Weber et D. Mohr. -N1: 10, W3: 25, W4: 25, W9: 28, W10: 14, W11: 16, W12: 27, M15: 33, 36, M17: 8, 36,C18: 20, C19: 20, C20: 8, C23: 32, C25: 21, E27: 36, E28: 36, E29: 36, E30: 36, E32: 8, E34: 9, 30, E35: 32, E36: 32,; E37: 23, 27, 32, 34, S38: 27, 32, 36, S41: 16, 27, S42: 27, S45: 27, S48: 27, S50: 27, S53: 27 , S56: 27,15 .

— japonicum Lindb. - W10: 14, W11: 16, Error: 30.

Cnestrum schistii (F. Weber et D. D. Mohr) J. I. Hagen - C18: 20.

Codriophorus brevisetus (Lindb.) Bednarek-Ochyra et Ochyra-W10: 36, M15: 36, E29: 36, S41: 36, S44: 36, S55: 36.

- carinatus (Cardot) Bednarek-Ochyra et Ochyra W10: 2 .

- corrugatus Bednarek-Ochyra et Ochyra - W10: 36 , M15: 36, E29: 36, E34: 3, E36: 3, S41: 36, S44: 36.

- fascicularis (Hedw.) Bednarek-Ochyra et Ochyra E29: 36, ?E37: 8, S55: 15, Error: 14, 16, 22, 27, 33.

Conostomum tetragonum (Hedw.) Lindb. $-\mathrm{N} 1:$ 10, M17: 36, E29: 36, E37: 4, S55: 15.

Coscinodon cribrosus (Hedw.) Spruce - W10: 14.

Cratoneuron filicinum (Hedw.) Spruce-N1: 10, W10: 14, C21: 37, C25: 21, E28: 36, E34: 9, 30, E37: 37, S38: 27, S41: 16, S54: 15, S55: 15 .

*?Ctenidium molluscum (Hedw.) Mitt. - E37: 34.

Cynodontium asperifolium (Lindb. et Arnell) Paris E26: 36 .
- strumiferum (Hedw.) Lindb. - W9: 28, M15: 36, C18: 20, E26: 36, E29: 36, S41: 16, S43: 27, S55: 15 .

— tenellum (B.S.G.) Limpr. - M13: 36, C18: 20, E26: 36.

*Cyrtomnium hymenophyllum (B.S.G.) Holmen M16: 29.

Dichelyma falcatum (Hedw.) Myrin - M17: 1, E37: 37.

Dichodontium pellucidum (Hedw.) Schimp. - W9: 28, M17: 8, C25: 21, E27: 36, E29: 36, E33: 36, S41: 16, S54: 15 .

Dicranella cerviculata (Hedw.) Schimp. - N1: 10, W6: 25, 33, W9: 28, W10: 14, W12: 27, M15: 36, S41: 16, S44: 22, S55: 15 .

— crispa (Hedw.) Schimp. - C18: 20, C19: 20.

- heteromalla (Hedw.) Schimp. - W6: 33, M15: 33, S55: 15 .

— palustris (Dicks.) Crundw. - W10: 14, M15: 36, C25: 21, E27: 36, S41: 27, S44: 8, 22, S46: 27, S51: 36, S55: 15 .

- *schreberiana (Hedw.) H.A. Crum et L. E. Anderson-E37: 4.

- subulata (Hedw.) Schimp. - N1: 10, C19: 20, C25: 21, E26: 36, E29: 36, S41: 16, S44: 22, S55: 15, Error: 33

- varia (Hedw.) Schimp. - W11: 16.

Dicranodontium denudatum (Brid.) E. Britton -W9: 28, W10: 14, M17: 8, S41: 16.

Dicranum acutifolium (Lindb. et H.Arnell) C.Jens. ex J. G. J. G. Weinm. - N1: 10, C21: 29, C25: 21, E29: 36, E31: 36, E34: 30, S55: 15 .

- bergeri Blandow in Starke - N1: 10, W6: 33, W10: 14, W11: 16, W12: 27,_M13: 36, M17: 8, 36, C19: 20, C25: 21, E34: 9, 30, 36, E37: 29, S41: 16, S42: 27, S52: 27, S55: 15.

- bonjeanii De Not. - W10: 14, M15: 33, 36, C18: 20, C19: 20, C25: 21 , E27: $36, \mathbf{E 2 9}: 36, \mathbf{E 3 0}: 36$, E34. 30, 36, E37: 36, S41: 16, S51: 36, S55: 15.

- brevifolium (Lindb.) Lindb. - N1: 10, M15: 36, C18: 20, C19: 20, E26: 36, E27: 36, E29: 36, E31: 36, E34: 30, E37: 37, S41: 36, S55: 15.

- elongatum Schleich. ex Schwaegr. - N1: 33, 10, W6: 33, W12: 27, M13: 36, E29: 36, E31: 36, E32: 8, E34: 30, 36, S41: 16, S43: 27, S55: 15.

- flagellare Hedw. - E26: 36, E30: 36.

- flexicaule Brid. - S46: 27, C25: 21.

- fragilifolium Lindb. - W8: 25, W9: 28, W10: 14, W11: 16, M17: 36, C20: 29, 36, E26: 36, E27: 36, E30: 36, E32: 29, E34: 9, S41: 16, S51: 36, S55: 15.

- fuscescens Turner - N1: 33, 10, W3: 25, W4: 25 , W10: 14, W11: 16, W12: 27, M15: 36, M17: 36, C18: 20, C19: 20, C20: 36, C21: 27, C25: 21, E26: 8, 36, E29: 36, E30: 36, E31: 36, E33: 36, E34: 30, E36: 36, E37: 23, 27, S41: 16, S45: 27, S46: 27, S47: 27, S49: 27, S55: 15 . 
_ * groenlandicum Brid. - S54: 29, Error: 27.

— japonicum Mitt. - W11: 36, C25: 21, Error: 30.

- laevidens R. S. Williams - E26: 36.

- leioneuron Kindb. - M15: 33, E29: 36, E31: 36, S41: 16.

— majus Sm. var. majus - N1: 10, W9: 36, W10: 14, W11: 16, W12: 27, 25, M14: 36,M15: 33, 36, M17: 36, C18: 20, C19: 20, C20: 36, C22: 27, C25: 21, E26: 8, 36, E27: 36, E29: 36, E30: 36, E33: 36, E34: 9, 30, E37: 27, S41: 16, S42: 27, S43: 27, S46: 27, S49: 27, S51: 36, S53: 27, S54: 15, S55: 15, S56: 15, 27.

- majus Sm. var. orthophyllum A. Braun ex MildeW9: 36, W10: 14, W11: 16, M13: 36, E29: 36, S41: 16, S51: 36 .

— montanum Hedw. - W9: 28, W10: 14, W11: 16, M15: 33, M17: 36, C18: 20, C19: 20, C20: 29, 36, C21: 29, C25: 21, E26: 36, E27: 36, S41: 16, S51: 36, S53: 29.

_ *?muehlenbeckii B.S.G. - W2: 25, M15: 33, Error: 29.

— polysetum Sw. - M15: 33, 36, M17: 36, C18: 20, C20: 29, 36, C21: 27, E26: 36, E30: 36,E34. 30, E37: 4, 29.

- scoparium Hedw. - W3: 25, W10: 14, W11: 16, M15: 36, C25: 21, E29: 36, E34: 9, 30, E36: 27, E37: 27, 34, 35, S39: 8, S40: 27, S42: 27, S45: 27, S48: 27, S52: 27, S55: 15.

- spadiceum J. E. Zetterst. var. spadiceum - N1: 33, W6: 33, C25: 21, E29: 36, E31: 36, E34: 9, M15: 36, S55: 15, Error: 27.

- spadiceum J. E. Zetterst. var. subscabrifolium Schljakov - E29: 36.

- spurium Hedw. - W7: 25, S41: 16.

_ *tauricum Sapehin - C21: 29, S53: 27.

Didymodon asperifolius (Mitt.) H. A. Crum et al. E31: 36.

— icmadophilus (Schimp. ex Muell. Hal.) K. Saito E29: 36.

- gaochienii B.C. Tan et Y. Jia - W9: 28.

- peroptusus Broth. - E29: 36.

Diphyscium foliosum (Hedw.) Mohr - W10: 14.

Distichium capillaceum (Hedw.) B.S.G. - N1: 10, W5: 25, W10: 14, M15: 33, M16: 29, M17: 8, 36, C18: 20, C25: 21, E27: 36, E29: 36, E32: 8, E34: 9, E37: 34, S51: 36, S55: 15.

— *inclinatum (Hedw.) B.S.G. - E34: 9.

Ditrichum cylindricum (Hedw.) Grout - N1: 10, W9: 25, W10: 14, W11: 16, C18: 20, S41: 16, S44: 22.

- flexicaule (Schwaegr.) Hampe - N1: 33, M16: 29, C18: 20, C22: 29, E27: 36, E29: 36, E31: 36, S41: 16, S55: 15 .

—*heteromallum (Hedw.) E. Britton - W6: 33.

- lineare (Sw.) Lindb. -W11: 16, E29: 36, S44: 22.

— pusillum (Hedw.) Hampe - C25: 21, S44: 22.
Drepanocladus aduncus (Hedw.) Wartnts. -N1: 10, M14: 36, M15: 33, 36, E27: 36, E28: 36, E34: 9, S44: 8 .

- arcticus (Williams) Hedenaes - S55: 15.

- polycarpus (Blandow ex Voit) Warnst. - W11: 16, C18: 20, C25: 21, E33: 36, S41: 16.

- polygamus (B.S.G.) Hedenaes - N1: 10, C22: 27, C25: 21, E27: 36, E28: 36, E29: 36, E34: 36.

_*sendtneri (Schimp. ex Muell. Hal.) Warnst. - E37: 27.

Echinophyllum sachalinensis (Lindb.) O'Brian - W3: 37, W5: 25, E27: 36, E34: 36, E37: 37.

Encalypta alpina Sm. - E29: 36.

_*iliata Hedw. - M17: 8.

- rhaptocarpa Schwaegr. -W10: 14, C18: 20, E29: 36, E31: 36, E32: 8, E34: 9.

— *vulgaris Hedw. - E37: 34.

Entodon concinnus (De Not.) Paris - M15: 36, E31: 36.

- rubicundus (Mitt.) A. Jaeger et Sauerb. - M15: 36.

Eurhynchiadelphus eustegius (Besch.) Ignatov et Huttonen - W10: 36.

Eurhynchiastrum pulchellum (Hedw.) Ignatov et Huttunen-C18: 20, C20: 8, C25: 21, E29: 36, E34.: 9, S41: 16 , S55: 15 , S56: 15.

Fissidens adianthoides Hedw. - C19: 20, S52: 27.

- bryoides Hedw. - M16: 29, C20: 8, C18: 20, E29: 36, S41: 16.

- dubius P. Beauv. -W10: 14,; S41: 16.

—osmundoides Hedw. - N1: 10; C25: 21, E29: 36, E31: 36 , S38: 1 .

- viridulus (Sw.) Wahlenb. - E29: 36.

*Fontinalis antipyretica Hedw. var. gracilis (Lindb.) Schimp. - W6: 33.

- antipyretica Hedw. var. antipyretica - W7: 25, W11: 16.

— hypnoides C. C. Hartm. -W12: 27, C25: 21, E34: 9.

Funaria hygrometrica Hedw. - N1: 10, W11: 16, M17: 8, C20: 36, C21: 32, C22: 32, C23: 27, 32, C25: 21, E27: 36, E28: 36, E33: 36, E34: 9, 32, E37: 34, 23, 32, S38: 36, S41: 16, S55: 15, S56: 15.

Grimmia alpestris (F. Weber et D. Mohr) Schleich. ex Nees. -E29: 24, S44: 22, S55: 15.

- anomala Hampe ex Schimp. - E28: 37.

_ *donniana Sm. - E37: 24, Error: 10.

- hartmanii Schimp. - W10: 14.

- incurva Schaegr. - E29: 24, S55: 15.

— longirostris Hook. - W10: 14, M15: 24, E29: 24.

— mollis Bruch et Schimp. - E29: 36, S55: 36.

_ *ovalis (Hedw.) Lindb. - M15: 33.

— reflexidens Muell. Hal. -C25: 21, E29: 24, S46: 27.

Helodium blandowii (F. Weber et D. Mohr) Warnst. N1: 33, W6: 33, W9: 36, W10: 14, W11: 16, M13: 36, M15: 33, C20: 36, C25: 21, E26: 36, E27: 36, E28: 36, E32: 27, E34: 9, E37: 23, 33, S56: 15, 27. 
Hennediella heimii (Hedw.) R.H. Zander var arctica (Lindb.) R.H. Zander - E29: 36.

*Herzogiella adscendens (Hedw.) Z. Iwats. et W.B. Schofield - E37: 29, M16: 29, S39: 8.

Heterocladium dimorphum (Brid.) B.S.G. - M15: 33, M17: 8, C25: 21, E34: 36, E35: 37.

*?Homalothecium lutescens (Hedw.) Robins. - E37: 34, S48: 27.

Hygroamblystegium humile (P. Beauv.) Vanderpoorten -W9: 28, W10: 14, S56: 27.

— *tenax (Hedw.) Jenn. - M17: 8.

Hygrohypnella duriuscula (De Not.) Ignatov et Ignatova-N1: 10, W10: 14, E28: 36, S41: 16, S44: 22.

- ochracea (Turner ex Wilson) Ignatov et Ignatova N1: 5, 10, W10: 14, W11: 16, M14: 36, M17: 36, C18: 20, C20: 8, C25: 21, E33: 36, E37: 29, S38: 27, S41: 16, S42: 27, S44: 8, 22, S55: 15.

Hygrohypnum bestii Renauld \& Bryhn $-\mathrm{N1}$ : 13, W10: 14, C18: 20, E27: 36, E28: 36, E36: 13, E37: 13, S55: 13 .

— luridum (Hedw.) Jenn. - M17: 36, C18: 20, C25: 21.

Hylocomiastrum pyrenaicum (Spruce) M. Fleisch. W9: 28, W10: 14,; W11: 16, M15: 36, M16: 29, M17: 8, C25: 21, E27: 36,E29: 36, E34: 36, S41: 16, S55: 15.

Hylocomium splendens (Hedw.) B.S.G. var. obtusifolium (Geh.) Paris - M17: 8, E29: 36, E31: 36.

- splendens (Hedw.) B.S.G. var. splendens - N1: 10, W10: 14, W11: 16, M17: 8, 36, M15: 33, 36, C18: 20, C19: 20, C20: 36, E27: 36, E29: 36, E30: 36, E32: 27, E34: 9, E37: 34, S41: 16, S51: 36, S55: 15, S56: 15 .

Hymenoloma crispulum (Hedw.) Ochyra - N1: 10, W10: 14, M14: 36, M15: 33, 36, M17:8, C25: 21, E26: 36, E27: 36 , E29: 36 , S41: 16, S44: 22, S51: 36, S55: 15 .

— intermedia (J. J. Amman.) Ochyra-E29: 36, S55: 15.

Hypnum cupressiforme Hedw. - W10: 14, M17: 36, C18: 20, E27: 36, E29: 36, E34: 30, E37: 29 , 34, S51: 36 .

— *?imponens Hedw. - W5: 25.

Isopterygiopsis alpicola (Lindb. \& Arnell) Hedenaes -C18: 20, S41: 16.

- muelleriana (Schimp.) Z. Iwats. -W10: 14, E27: 36, E29: 36, E32: 8.

- pulchella (Hedw.) Z. Iwats. - M13: 36, M16: 29, C18: 20, C25: 21, E29: 36, E34: 9, E37: 34.

*? Isothecium alopecuroides (Dubois) Isov. - E37: 34.

Iwatsukiella leucotricha (Mitt.) W. R. Buck et H. A. Crum - W9: 28, W10: 14, W11: 16.

Kiaeria blyttii (Schimp.) Broth. -S44: 22, S55: 15.

- falcata (Hedw.) J. I. Hagen - S44: 22, S55: 15.

— glacialis (Berrg.) J. I. Hagen - M15: 36, E29: 36, S43: 27.
- starkei (F. Weber et D. Mohr) J. I. Hagen - E29: 36, S41: 16, S44: 8, 22, S46: 27, S55: 15,

Leptobryum pyriforme (Hedw.) Wilson - N1: 10, W4: 25, W11: 16, M15: 36, M17: 36, C18: 20, C19: 20, C20: 8, 36, C22: 27, E26: 36, E27: 36, E28: 36, E29: 36, E30: 36, E33: 36, E34: 9, E37: 34,S38: 36, S41: 16, S44: 22, S55: 15.

Leptodictyum riparium (Schimp.) Warnst. -N1: 33, W10: 14, E28: 36, S44: 22, S55: 15, S57: 27.

Lescuraea mutabilis (Brid.) Lindb. -W10: 14, M15: 33.

- incurvata (Hedw.) Lawt. - N1: 10, M15: 33, C25: 21, E37: 34 .

- patens (Lindb.) Arnell et C. Jens. - N1: 10, M14: 36, C18: 20, S55: 15.

- radicosa (Mitt.) Moenk. -W10: 14, S55: 15.

- secunda Arnell-C25: 21, S38: 27, S45: 27.

Leskea polycarpa Hedw. - N1: 10, W10: 14, C25: 21, S44: 8 .

*Limprichtia cossonii (Schimp.) L. E. Anderson et al. -N1: 33, E37: 27.

- revolvens (Sw.) Loeske - N1: 10, M17: 36, E29: 36, E31: 36, E34:36, E36: 33, E37: 33, S51: 36,S55: 15, S56: 15.

*Loeskeobryum brevirostre (Brid.) M. Fleisch. in Broth. - S45: 27.

Loeskypnum badium (C. C. Hartm.) Paul. - M17: 8, E29: 36, E31: 36, E37: 33.

*Meesia longiseta Hedw. - Kabiersch, 1937 (1938) without location.

- triquetra (Richter) Aongstr. - E34: 36, E36: 33, S51: 36, S55: 15, S56: 15.

— uliginosa Hedw. - M17: 36, E29: 36, E31: 36, E37: 4, 34 .

Mnium laevinerve Cardot - W11: 16, S41: 16. This species was separated from $M$. lycopodioides by Savicz-Lyubitskaya \& Smirnova (1970), Noguchi (1989), and Koponen (1981). Later Koponen (1994) combined them, due to numerous transitional specimens. In Kamchatkan material these taxa however are distinct, differeing primarily in cells size (15-20 $\mathrm{mm}$ in M. laevinerve, $20-30 \mathrm{~mm}$ in M. lycopodioides), and also in always smooth costa in M. laevinerve.

- lycopodioides Schwaegr. (incl. M. ambiguum $\mathrm{H}$. Muell.) - W9: 28, W10: 14, S41: 16. Czernyadjeva \& Potemkin (2003) treated M. ambiguum as a separate species, but additional collections convinced us that these species are unseparable. Thus we follow Koponen (1994) who combined them.

- marginatum (Dicks.) P. Beauv. - C20: 8, C25: 21.

- spinosum (Voit.) Schwaegr. -W10: 14, M15: 33, S41: 16 .

- stellare Hedw. - C18: 20, C25: 21.

Myurella julacea (Schwaegr.) B.S.G.-W10: 14, M15: 
33, E29: 36, E31: 36, E34:9, S51: 36.

- tenerrima (Brid.) Lindb. - E31: 36, E32: 8.

- sibirica (Muell. Hal.) Reimers - C25: 21, E31: 36.

Myuroclada maximowiczii (Borszcz. in Maxim.) Steere et W. B. Schofield - W10: 14, S41: 16.

Neckera pennata Hedw. - E37: 34, M5: 36.

Niphotrichum barbuloides (Cardot) Bednarek-Ochyra et Ochyra - W10: 36.

- canescens (Hedw.) Bednarek-Ochyra et Ochyra N1: 10, W7: 25, W10: 14, M15: 33, 36, M17: 8, C25: 37, C25: 21, E26: 8, 36, E29: 36, E31: 36 , E32: 8, E33: 36, E34: 9, E35: 37, E37: 27, 36, S38: 37, S39: 27, S41: 16, S44: 22, S55: 15.

- canescens var. latifolium (C. E. O. Jensen) Bednarek-Ochyra et Ochyra - W10: 36.

- ericoides (F. Weber ex Brid.) ) Bednarek-Ochyra et Ochyra-W10: 36, C25: 21, E29: 36, E37: 27, S41: 27, S42: 27, S44: 22.

- muticum (Kindb.) Bednarek-Ochyra et Ochyra S44: 22, S55: 15.

- panschii (Muell. Hal.) Bednarek-Ochyra et Ochyra - E29: 36.

Ochyraea cochlearifolium (Venturi ex De Not.) Ignatov et Ignatova - E29: 36.

Oligotrichum aligerum Mitt. -W11: 19, C25: 19.

- hercynicum (Hedw.) DC. -W12: 8, M17: 8, C25: 21, E29: 36, S44: 8, 22, S55: 15.

— parallelum (Mitt.) Kindb. - W10: 14, M14: 36, M15: 33, C25: 21, E27: 36, S39: 8, S41: 16, S44: 22, S46: 27, S55: 15.

Oncophorus compactus (B.S.G.) Schljakov - E29: 36, E31: 36.

_ crispifolius (Mitt.) Lindb. - W9: 28, W10: 14, W11: 16.

— wahlenbergii Brid. - N1: 33, 10, W9: 36, M17: 36 , C18: 20, C20: 8, 36, E26: 36, E29: 36, E31: 36, E33: 36, E34: 36, E37: 29, 34, S41: 16, S55: 15, S56: 15.

— virens (Hedw.) Brid. var. virens -N1: 5, E29: 36, E31: 36, E34: 30, Error: 10, 15.

- virens var. serratus (B.S.G.) Braithw. - E29: 36.

Orthothecium chryseum (Schwaegr.) B.S.G. - M16: 29, E31: 36.

*?Orthotrichum affine Brid. - E37: 34.

—obtusifolium Brid. -W9: 28, M13: 36, M17: 8, 36, C18: 20, C19: $20, \mathbf{C 2 0}: 8, \mathbf{C 2 5}: 21, \mathbf{E 2 7}: 36, \mathbf{E 2 8}$ : 36, E37: 29, S41: 16.

— *pylaisii Brid. - M17: 8.

— sordidum Sull. et Lesq. - W9: 28, W10: 14, W11: 16, M13: 36, M17: 8, C18: 20, C19: 20, C25: 21, E26: 36, E28: 8, E28: 36, E37: 8, S41: 16, S51: 36 .

— * *speciosum Nees in Sturm - M15: 33.

Oxystegus tenuirostris (Hook. et Taylor) A.J.E. Smith - N1: 10, W10: 14, M15: 36, C20: 8, C25: 21.

Oxyrrhynchium hians (Hedw.) Loeske - W9: 28.

Paludella squarrosa (Hedw.) Brid. - N1: 5, 33, W9:
36, W10: 14, W11: 16, M13: 36, M14: 36, M15: 33, 36, M17: 1, C25: 21, E31: 36, E34: 9, 30, E36: 33, E37: 8, S45: 27, S56: 15 .

*Palustriella commutata (Hedw.) Ochyra-E37: 4, 34. Paraleucobryum enerve (Thed. in Hornem.) Loeske E29: 36.

- longifolium (Hedw.) Loeske - W9: 28, W10: 14, M15: 33, C25: 21, E37: 29, S41: 16.

Philonotis caespitosa Jur. -N1: 10, W12: 27,C25: 21, S41: 16, S44: 8, S45: 27, S55: 15.

- fontana (Hedw.) Brid. - N1: 10, W8: 25, M15: 33, 36, C25: 21, E33: 36, E34: 9, 36, E36: 33, S44: 22 , S47: 27, S55: 15 .

- tomentella Molendo - N1: 5, 10, W10: 14, W11: 16, M14: 36, C18: 20, C25: 21, E29: 36, E31: 36, E33: 36, E34: 36, S41: 16, S44: 22, S45: 27, S46: 27, S51: 36 , S55: 15 .

- yezoana Besch. et Cardot ex Cardot-S44: 12, 22, S55: $12,15$.

Plagiomnium acutum (Lindb.) T. J. Kop. - W8: 25, W9: 25, 28, W10: 14, W11: 16, M15: 33, M17: 8, C18: 20, E28: 8, E37: 8, 27, S41: 16.

- affine (Blandow) T. J. Kop. - W10: 14, W12: 27, C25: 21, S53: 27, S56: 27.

— curvatulum (Lindb.) Schljakov - N1: 10, W11: 16, M17: 8 .

- cuspidatum (Hedw.) T. J. Kop. - W9: 28, W11: 16, C18: 20, C25: 21, E28: 8, E28: 36, E34: 9, E37: 27, 35, S41: 16 .

- drummondii (Bruch \& Schimp.) T. J. Kop. - S41: 16.

— *elatum (B.S.G.) T. J. Kop. - C22: 27.

- ellipticum (Brid.) T. J. Kop. - N1: 10, W9: 36, W10: 14, W11: 16, M15: 33, 36, M17: 8, C18: 20, C25: 21,E28: 36, E37: 33, S55: 15, S56: 15.

- medium (Bruch et Schimp.) T. J. Kop. - W9: 28, W10: 14, W11: 16, M15: 33, 36, C18: 20, C19: 20, C21: 27, C25: 21, E37: 8, S41: 16, S55: 15 .

— ${ }^{*}$ rostratum (Schrad.) T. J. Kop. - E37: 23, S48: 27.

* Plagiopus oederiana (Sw.) H.A. Crum et L. E. Anderson - E37: 23.

Plagiothecium cavifolium (Brid.) Z. Iwats. var. cavifolium-W9: 28, W10: 14, M17: 8, W11: 16, C18: 20, C20: 8, C25: 21, E27: 36, E29: 36, E32: 8, E37: 29, S41: 16 , S51: $36, \mathbf{S 5 5}: 15$.

- cavifolium (Brid.) Z. Iwats. var. imbricatum Ukrainskaya-W10: 14.

- denticulatum (Hedw.) B.S.G. - N1: 10, W9: 28, W10: 14, W11: 16, C18: 20, C25: 21, E27: 36, E29: 36, E34: 9, 36, E37: 23,29, 36, M15: 33, 36, M17: 8, 36, S39: 8, S41: 16, S44: 22, S45: $27, \mathbf{S 4 6 :} 27$, S53: 27, S53: 27, S55: 15 , S56: 15.

- euryphyllum (Cardot et Ther.) Z. Iwats. -W11: 16, S41: 16, S55: 15.

- laetum Schimp. - N1: 10, W3: 25, W9: 28, W10: 
14, W11: 16, M15: 36, M17: 36, C19: 20, E34: 9, 36, S41: 16, S51: 36, S55: 15, C18: 20.

- latebricola B.S.G. - W9: 28, C25: 21, S41: 16.

- nemorale (Mitt.) A. Jaeger - W9: 28, W10: 14, W11: 16, M15: 36, C25: 21, E27: 36, E37: 29, S41: 16, S46: 27, 29, S55: 15.

— *?plathyphyllum Moenk. - M15: 33, S45: 29.

Platydictya jungermannioides (Brid.) H. A. Crum C25: 21.

Platygyrium repens (Brid.) B.S.G. - W9: 28, W10: 14, W11: 16, C25: 21, S41: 16.

Pleuridium subulatum (Hedw.) Rabenh. - W10: 14, W11: 16.

Pleuroziopsis ruthenica (J. G. Weinm.) Kindb. ex E. Britton-W7: 25, W10: 14, W11: 16, E37: 32, S41: 16.

Pleurozium schreberi (Brid.) Mitt. - N1: 10, W6: 33, W9: 28, W10: 14, W11: 16, M13: 36, M15: 33, 36, M17: 8, 36, C20: 36, C25: 21, E26: 36, E27: 36, E29: 36, E30: 36, E31: 36, E34: 9, 30, E37: 34, S41: 16 , S51: 36 , S55: 15 , S56: 15.

Pogonatum contortum (Brid.) Lesq. - W10: 14, W11: 16, E37: 23 .

- dentatum (Brid.) Brid. -W6: 33, W10: 14, W11: 16, W12: 8, C19: 20, C23: 32, C25: 21, E26: 8, E29: 36, E34: 9, E36: 32, S41: 16, S43: 8, S55: 15.

— japonicum Sull. et Lesq. - W11: 16, C20: 7, E34: 7, 9, 30, E37: 7, 32, S55: 15 .

- urnigerum (Hedw.) P.Beauv. - N1: 10, W12: 8, C19: 20, C25: 21, E27: 36, E28: 8, E29: 36, E31: 36, E34: 9, 36, E37: 4, 8, 27, 32, 36, S38: 8, S44: 22.

Pohlia andalusica (Hoehnel) Broth. -E31: 36, C25: 21, S44: 22.

— andrewsii A.J. Shaw - E29: 36, E32: 8, S41: 16.

— annotina (Hedw.) Lindb. -W10: 14, C25: 21, S41: 16, S44: 22, S55: 15.

— bulbifera (Warnst.) Warnst. - N1: 10, S41: 16.

- cardotii (Renauld et Cardot) Broth. -S44: 22, 11, S55: 15,11 .

- cruda (Hedw.) Lindb. - N1: 10, W10: 14, M15: 33, 36, M17: 8, 36, C18: 20, C19: 20, C20: 8, C25: 21, E26: 8, 36, E27: 36, E28: 36, E29: 36, E30: 36, E32: 8, E34: 9, E37: 27, 34, S39: 8, S41: 16, S51: 36, S55: 15 .

— crudoides (Sull. et Lesq.) Broth. - W10: 14, M15: 36, C25: 21, E29: 36, E31: 36, S41: 16, S55: 15.

- drummondii (Muell. Hal.) A. L. Andrews -N1: 10, M14: 36, C25: 21, E29: 36, E31: 36, S41: 16, S44: 22, S46: 29, S55: 15 .

— filum (Schimp.) Mart. - N1: 10, W10: 14, C25: 21, E26: 36, E29: 36, E33: 36, E37: 36, S44: 22, S55: 15.

- longicollis (Hedw.) Lindb. -W10: 14, M17: 8, E32: 8, S41: 16.

— nutans (Hedw.) Lindb. - N1: 10, W6: 33, W9: 28,
W10: 14, W11: 16, W12: 27, M15: 36, M17: 8,36 , C18: 20, C19: 20, C20: 36, C25: 21, E26: 8, 36, E27: 36,E28: 36, E29: 36, E30: 36, E31: 36, E32: 8, E33: 36, E34: 9, 30, E37: 4, 23,27, S41: 16, S43: 27, S44: 22, S46: 27, S51: 36 , S54: 15 , S55: 15 , S56: 15 .

— proligera (Lindb. ex Breidl.) Lindb. ex Arnell - W9: 28, W10: 14, W11: 16, C18: 20, C25: 21, C25: 21 , E29: 36, S38: 36, S41: 16, S46: 29, S51: 36.

- tundrae A. J. Shaw - W11: 18, C18: 18.

- wahlenbergii (F. Weber et D. Mohr) A.L. Andrews - N1: 10, W9: 28, M14: 36, M15: 36, M17: 36, C18: 20, C22: 27, C25: 21 , E27: 36, E28: 36, E29: 36, E33: 36, E34: 9, 36, E37: 36, S41: 16, S43: 27 , S44: 8, 22, S55: 15, Error: 33 .

Polytrichastrum alpinum (Hedw.) G.L.Sm. - N1: 5, 10, W6: 33, W9: 28, W10: 14, W11: 16, W12: 8, M14: 36, M15: 33, M17: 8, C18: 20, C19: 20, C20: 8, C23: 32, C25: 21, E26: 8, E27: 36, E28: 8, E29: 36, E31: 36, E33: 36, E34: 9, 30, 32, E35: 32, E36: 32, E37: 8, 23, 27, 32, S38: 32, S39: 8, S40: 27, S41: 16, S44: 22, S45: 27, S46: 27, S52: 27, S53: 27, S55: 15, S56: 27.

- formosum (Hedw.) G.L.Smith - W10: 14, E37: 35.

- longisetum (Sw. ex Brid.) G.L.Smith - N1: 10, W10: 14, W11: 16, W12: 27, M15: 36, C18: 20, C19: 20, C25: 21, E27: 36, E34: 9, E37: 8, 32, S41: 16, S45: 27, S53: 27, S56: 27.

— *norvegicum (Hedw.) Schljakov- E34: 9, E37: 27, S56: 27, S57: 27.

- sexangulare (Floerke ex Brid.) G.L.Smith - N1: 5, M14: 36, M15: 36, C25: 21, E29: 36, E32: 8, E34: 9, 32, E37: 4, S41: 16, S44: 8, 22, S55: 15.

- sphaerothecium (Besch.) Schljakov-E31: 36, E34: 9.

Polytrichum commune Hedw. - N1: 5, 33, W6: 10, 33, W9: 36, W10: 14, W11: 16, M15: 36, M17: 8, 36, C18: 20, C19: 20, C20: 8, C20: 36, C21: 27, 32, C22: 27, C23: 32, C25: 21, E26: 36, E27: 36, E28: 36, E29: 36, E30: 36, E34: 9, 30, E35: 32, E36: 32, E37: 4, 23,27, 32, 34, S38: 32, S41: 16, S42: 27, S43: 27, S44: 22, S45: 27, S46: 27, S47: 27, S49: 27, S54: 15 , S55: 15 .

- hyperboreum $\mathrm{R}$. Br. -N1: 10, W3: 36, M13: 36, E26: 36, E34: 30, E37: 36, S55: 15.

- jensenii I. Hagen -W6: 33, W10: 14, W12: 27, M15: 33, 36, S44: 22, E34: 9, Error: 30.

- juniperinum Hedw. - N1: 33, 10, W6: 33, W9: 28, W10: 14, W11: 16, M15: 33, M17: 36, C18: 20, C19: 20, C20: 8, 36, C21: 32, C23: 32, C24: 32 , C25: $21,32,36$, E26: $36, \mathbf{E 2 7}: 36$, E28: 8,36 , E29: 36, E30: 36, E31: 36, E32: 8, E33: 36, E34: 9, 30, 32, E36: 27, 32, E37: 4, 27, 32, 34, 35, S38: 32, 36, S41: 16, S43: 27, S44: 22, S48: 27, S49: 27, S52: 27, S54: 15, S55: 15 . 
— piliferum Hedw. -N1: 10, M14: 36, M15: 36, M17: 8, 36, C18: 20, C20: 8, 36, C25: 21, 32, E26: 8, 36, E27: 36, E29: 36, E30: 36, E32: 8, E33: 36, E34: 9, 30, 32, E35: 32, E36: 32, E37: 8, 27, 32, 36, S38: 32, S41: 16, S43: 8 , S44: 8 , 22, S46: 27, S51: 36, S55: 15 .

- strictum Brid. - N1: 10, 33, W5: 32, W6: 33, W10: 14, W12: 27, M13: 36, C25: 21, E27: 36, E29: 36, E34: 9, 30, 32,E35: 32, E36: 27, 32, E37: 32, S41: 16, S42: 27, S55: 15.

— *?swartzii C. C. Hartm. - W2: 25.

Pseudobryum cinclidioides (Huebener) T. J. Kop. N1: 10, W8: 25, W9: 28, W10: 14, W11: 16, M15: 36, C25: 21, E27: 36, E34: 9, 36, S41: 16, S45: 27, S51: 36, S56: 15 .

*?Pseudocalliergon lycopodioides (Brid.) Hedenaes N1: 33 .

- turgescens (T. Jensen) Loeske - E31: 36.

Pseudoleskeella nervosa (Brid.) Nyh. - W9: 28, W10: 14, W11: 16, M15: 33, C18: 20, E27: 36, E28: 36, S38: 8, S41: 16, S51: 36.

— papillosa (Lindb.) Kindb. - E29: 36.

- tectorum (Funck ex Brid.) Kindb. in Broth. - C18: 20.

Pseudotaxiphyllum elegans (Brid.) Z. Iwats. - W10: 14, S55: 15.

Psilopilum laevigatum (Wahlenb.) Lindb. - N1: 5, S41: 16.

Pterigynandrum filiforme Hedw. - W10: 14, S51: 36.

Ptilium crista-castrensis (Hedw.) De Not. - N1: 10, W5: 25, W10: 14, M17: 36, C18: 20, C22: 27, S41: 16, S46: 27, S55: 15.

Pylaisia polyantha (Hedw.) B.S.G. - W9: 28, W10: 14, W11: 16, M15: 33, M17: 36, C18: 20, C19: 20, C20: 27, C25: 21, E26: 36, E28: 36, E34: 9, E37: 8, 34, S41: 16.

- selwynii Kindb. - W9: 28, S41: 16.

- subcircinata Cardot - W9: 28, W10: 14, W11: 16, C18: 20, C19: 20, S41: 16.

Racomitrium lanuginosum (Hedw.) Brid. - N1: 10, W10: 14, M13: 36, M17: 8, 36, E26: 36, E29: 36, E31: 36, E32: 8, E34: 9, 30, E36: 37, E37: 4, 36, S41: 27, S44: 22, S51: 36 , S54: 15 , S55: 15.

Rauiella fujisana (Paris) Reimann - W9: 28, W11: 16.

*Rhabdoweisia crispata (Dicks.) Lindb. - M17: 8.

Rhizomnium andrewsianum (Steere) T. J. Kop. - S55: 15.

- gracile T. J. Kop. - C18: 20, S41: 16.

- magnifolium (Horik.) T. J. Kop. -N1: 10, W3: 25, W9: 28, W10: 14, W11: 16, M15: 36, C18: 20, C25: 21, E27: 36, S41: 16, S56: 15.

- nudum (E. Britton et R. S. Williams) T. J. Kop. N1: 5, W9: 28, W10: 14, W11: 16, M15: 33, 36, C25: 21, E34: 9, E35: 6, E37: 29, S39: 8, S41: 16, S44: 22, Error: 30.
- pseudopunctatum (Bruch et Schimp.) T. J. Kop. N1: 10, W9: 28, W10: 14, C18: 20, E34: 36, S51: 36, S55: 15.

— punctatum (Hedw.) T. J. Kop. - C25: 21, E37: 23,27, S42: 27, S45: 27, S56: 27.

- striatulum (Mitt.) T. J. Kop. - W9: 28, S41: 16.

Rhodobryum roseum (Hedw.) Limpr. - W5: 25,W9: 28, W10: 14, W11: 16, M15: 33, 36, C18: 20, C19: 20, E27: 36, E30: 36, E34: 9, 30, S41: 16, S42: 27 , S45: 27, S51: 36, S54: 27.

Rhytidiadelphus squarrosus (Hedw.) Warnst. - N1: 10, W6: 33, W9: 28, W10: 14, W11: 16, M15; 33, 36, M17: 8, C25: 21, E30: 36, E34: 36, E37: 34, S41: 16, S42: 27, S45: 27, S48: 27, S50: 27, S53: 27 , S55: 15, S56: $15,27$.

- subpinnatus (Lindb.) T. J. Kop. - N1: 10, W3: 25, W4: 25, W5: 25, W8: 25, W10: 14, W11: 16, E27: 36, E35: 32, E37: 32, S41: 16, S45: 27, S52: 27 , S55: 15 .

-triquetrus (Hedw.) Warnst. - W6: 33, W10: 14, W12: 27, E27: 36, E37: 27, 32, 34,S51: 36, S55: 15, S56: 15.

Rhytidium rugosum (Hedw.) Kindb. - N1: 10, W10: 14, M15: 33, M17: 8, 36, C18: 20, C20: 36, C21: 32, E27: 36, E29: 36, E30: 36, E31: 36, E34: 32, 36, E37: 32, S51: 36, S52: 27, S55: 15.

Rigodiadelphus robustus (Lindb.) Nog. - W10: 14, W11: 16, S41: 16.

Saelania glaucescens (Hedw.) Brid. - M13: 36, M15: 33, M17: 1, C18: 20, C20: 8, E29: 36, E31: 36.

Sanionia uncinata (Hedw.) Loeske - N1: 10, 33, W2: 25, W3: 25, W9: 28, W10: 14, W11: 16, W12: 27, M13: 36, M14: 36, M15: 33, 36, M17: 8, 36, C18: 20, C19: 20, C20: $8,36, \mathbf{C 2 5}: 21$, E26: $8,36, \mathbf{E 2 7}$ : 36, E28: 36, E29: 36, E30: 36, E31: 36, E32: 8, 27 , E33: 36, E34: 9, 30, E37: 23, 27, 34, 35, 36, S38: 27, 36, S41: 16, S44: 8, 22, S45: 27, S46: $27, \mathbf{S 5 1}$ : 36, S54: 15, S55: 15, S56: 15, 27.

Schistidium agassizii Sull. et Lesq. - E31: 36 .

_ *?apocarpum (Hedw.) B.S.G. - M15: 33, M17: 8, E34: 30 . These records were made for this species sensu lato. All available collection however were reidentifyed to other species.

- dupretii (Ther.) W. A. Weber - E29: 36.

- frigidum H. H. Blom - W10: 36, E29: 36, E31: 36.

- maritimum (Turner) B.S.G. - S55: 15.

_papillosum Culm. -W10: 36, M15: 36, C18: 20, E29: 36.

- plathyphyllum (Mitt.) Perss. - C25: 21, E29: 36.

- pulchrum H. H. Blom - C25: 21.

- rivulare (Brid.) Podp. var. rivulare-W10: 14, C25: 21, E37: 4, S41: 16, S44: 22, S55: 15.

- rivulare (Brid.) Podp. var. latifolium (J. E. Zetterst.) H.A. Crum et L. E. Anderson - N1: 10, S55: 15.

- trichodon (Brid.) Poelt var. nutans Blom - M16: 39. 
Schistostega pennata Hedw. - W9: 28, C18: 20, C19: 20, E34.: 9, E35: 6, M17: 7, 8, 36.

Sciurohypnum glaciale var. dovrense (Limpr.) Ochyra - E29: 36.

- glaciale (B.S.G.) Ignatov et Huttunen var. glaciale -C25: 21, Error: 25, 27.

- latifolium (Kindb.) Ignatov et Huttunen - M16: 29, E29: 36, E31: 36, E34: 9, Error: 15.

- oedipodium (Mitt.) Ignatov et Huttunen - W9: 28, W10: 14, C18: 20, C19: 20, C25: 21, E26: 36, E29: 36, E33: 36, E34: 30, S41: 16, S44: 22.

- ornellanum (Molendo) Ignatov et Huttunen - W9: 28.

- plumosum (Hedw.) Ignatov et Huttunen s.l. - C18: 20, C20: 8, C25: 21, E29: 36, S41: 16, S51: 36.

- populeum (Hedw.) Ignatov et Huttunen - W2: 25 , C25: 21, E34: 30, E37: 23,S51: 36.

- reflexum (Starke) Ignatov et Huttunen - N1: 10, W3: 25, W4: 25, W9: 28, W10: 14, W11: 16, W12: 27, M15: 33, 36, M17: 36, C18: 20, C19: 20, C20: 36, C25: 21, E26: 36, E27: 36,E28: 36, E29: 36, E30: 36, E31: 36, E33: 36, E34: 9, 30, E36: 27, E37: 8, 27, 36, S38: 36, S39: 8, S40: 27, S41: 16, S42: 27, S43: 27, S44: 22, S45: 27, S46: 27, S51: 36, S53: 27, S54: 27, S55: 15, S56: 15.

- starkei (Brid.) Ignatov et Huttunen - N1: 10, W2: 25, W3: 25, W4: 25, W5: 25, W8: 25, W9: 25, 28, W10: 14, W11: 16, W12: 27, M15: 33, M17: 8 , C18: 20, C19: 20, C25: 21, E26: 36,E27: 36, E29: 36, S39: 8, S41: 16, S44: 22, S55: 15.

- unicifolium (Broth. et Paris) Ochyra et Zarnowiec - C18: 20, C25: 21, E27: 36.

Scorpidium scorpioides (Hedw.) Limpr.-N1: 33, E34: 30, E37: 33, S51: 1, S56: 15.

Sphagnum angustifolium (Russow) C. E. O. Jensen W9: 36, W10: 14, W11: 16, W12: 25, C23: 31, C25: 21, E34: 30, 31, E35: 31, E36: 31, E37: 31, S38: 31, S41: 16, S44: 22, S56: 15, 25.

- balticum (Russow) Russow ex C. E. O. Jensen C22: 31, E36: 31, S44: 22, S56: 15.

— capillifolium (Ehrh.) Hedw. - W10: 14, W11: 16, M15: 36, E29: 36, E34: 9, 31, S41: 16, S56: 15.

- centrale Arnell et C. E. O. Jensen - W10: 14, S38: 31, S53: 25, S55: 15, S56: 15.

- compactum DC. in Lam. et DC. - W10: 14, M17: 8, E29: 36, E34: 9, 31, 36, E36: 31, E37: 31, S41: 16, S51: 36 , S55: 15 .

- contortum Schultz - W10: 14, E36: 31, S41: 16.

- cuspidatum Ehrh. ex Hoffm. - W10: 14.

- $\operatorname{fallax}$ (H. Klinggr.) Isov. -W10: 14, W11: 16, W12: 25, 31, E36: 31, S38: 31, S44: 22.

- fimbriatum Wilson in Wilson et Hook. f.- W11: 16, W12: 25, 31, E34: 9, 30, 31, 36, E36: 31, S38: 31, S41: 16, S44: 22, S55: 15.

- flexuosum Dozy et Molk. - W11: 16.
- fuscum (Schimp.) H. Klinggr. - W5: 31, W9: 31, 36, W10: 14, W11: 16, W12: 25, C23: 31, E34: 9, 30, 31, E35: 31, E36: 31, E37: 31, S38: 31, S41: 16, S51: 36 .

— girgensohnii Russow - N1: 10, W8: 31, W9: 31, 36, W10: 14, M13: 36, M15: 36, M17: 8, 36, C22: 31, E29: 36, E32: 8, E34: 9, 30, 31, E35: 31, E36: 25, 31, E37: 31, S38: 31, S41: 16, S44: 22, S45: 25, S47: 25 , S56: 25 .

— *jensenii H. Lindb. - E34: 31.

- lindbergii Schimp. ex Lindb. - W5: 31, W10: 14, W11: 16, W12: 25, E34: 31, 30, 36, E36: 31.

- magellanicum Brid. - W10: 14, W11: 16, W12: 25, E34: 9, E37: 31, S38: 31, S51: 36, S56: 15.

— ${ }^{*}$ majus (Russow) S. Jens. - E34: 31, E36: 31, S38: 31.

_*obtusum Warnst. - S38: 31

— papillosum Lindb. W9: 36, W10: 14, W11: 16, E34: 30, 36, E37: 31, S38: 31.

— *platyphyllum (Lindb. ex Braithw.) Sull. ex Warnst. - E35: 31, E36: 31, E37: 31, S38: 31.

— ${ }^{*}$ pulchrum (Lindb.) Warnst. - W8: 31, E37: 31.

- riparium Aongstr. - W9: 36, W10: 14, W11: 16, W12: 25, E34: 9, 31, 36, E36: 31, E37: 25, 31, S38: 31, S44: 22, S46: 25, S55: 15 .

— rubellum Wilson-W9: 36, M13: 36, C22: 31, E34: 30, E36: 31, E37: 31, S38: 31.

- russowii Warnst. -W8: 31, W9: 36, W10: 14, W11: 16, E34: 30, 31, E36: 31, E37: 31, S38: 31, S41: 16, S44: 22, S46: 25.

- squarrosum Crome - W9: 36, W10: 14, M17: 8, C22: 31, E28: 36, E34: 9, 30, 31, E36: 31, E37: 23, 31, 35, S38: 31, 36, S46: 25, S56: 15, 25.

- subsecundum Nees - C25: 21, E34: 9, E36: 25, 31, E37: 25, 31, S38: 31, S46: 25.

— tenellum (Brid.) Bory - W10: 14, E36: 31, E37: 31, S38: 31 .

- teres (Schimp.) Aongstr. ex C. C. Hartm. - W10: 14, M15: 36, C23: 31, C25: 21, E34: 30, E35: 31, E36: 31, E37: 31, S38: 31, S42: 25, S46: 25, S53: 25, S56: 15 .

- warnstorfii Russow - W9: 36, W10: 14, M13: 36, M17: 8, C22: 31, C23: 31, C25: 21, E34: 9, 30, 31, E35: 31, E36: 31, E37: 31, S45: 25, S53: 25, S56: 15.

*Splachnum luteum Hedw. - E37: 4.

— *melanocaulon (Wahlenb.) Schwaegr. - E37: 34.

— *rubrum Hedw. - E37: 4.

- sphaericum Hedw. - N1: 5.

Stereodon bambergeri (Schimp.) Lindb. - E31: 36, Error: 8 .

- callichroum (Brid.) Braithw. - W10: 14, M16: 29, Error: 27, 30.

- holmenii (Ando) Ignatov et Ignatova-M13: 36.

— hamulosum (B.S.G.) Lindb. - E31: 36, S39: 8. 
— pallescens (Hedw.) Mitt. - N1: 10, W9: 28, W10: 14, W11: 16, E27: 36, S41: 16, S45: 27, S51: 36 , S55: 15 .

— plicatulum Lindb. -N1: 10, W7: 25, W9: 28, W10: 14, W11: 16, M15: 36, M17: 36, C18: 20, C19: 20, C25: 21, E27: 36, E29: 36, E34: 9, 36, S41: 16, S46: 29, S51: 36 , S55: 15 .

— revolutus (Mitt.) Lindb. - C18: 20, E29: 36.

_ *?subimponens (Lesq.) Broth. - Flora i rastitelnost... (2002), without locality.

- vaucheri (Lesq.) Lindb. et Broth. - C18: 20.

Straminergon stramineum (Dicks. ex Brid.) Hedenaes - N1: 10, 33, W9: 36, W10: 14, W11: 16, M13: 36, M15: 33, M17: 8, C25: 21, E34: 9, 30, S41: 16, 27, S44: 22, S45: 27, S46: 27, S55: 15.

Syntrichia norvegica F. Weber - W10: 14, M15: 36, C25: 21, E27: 36, E29: 36, S41: 16.

- ruralis (Hedw.) F. Weber \& D. Mohr-N1: 10, M15: 36, M17: 8, C18: 20, C25: 21, E28: 36, E29: 36, E37: 8, S41: 16, S51: 36, S55: 15, Error: 33.

Tayloria lingulata (Dicks.) Lindb. - N1: 5, M14: 36, S55: 15 .

- splachnoides (Scleich. ex Schwaegr.) Hook. - M15: 36.

Tetraphis pellucida Hedw. - W9: 28, W10: 14, W11: 16, M17: 8, 36, C18: 20, C19: 20, C20: 8, 36, E27: 36, E30: 36, S41: 16.

Tetraplodon angustatus (Hedw.) B.S.G. - C18: 20.

— mnioides (Hedw.) B.S.G. - N1: 10, W6: 33, W12: 27, M13: 36, E29: 36, E31: 36, E34: 9, 36, E37: 4, S43: 27 , S48:27, S55: 15.

— urceolatus (Hedw.) B.S.G. - M17: 8, E29: 36, E34: 36.

Thamnobryum alopecurum (Hedw.) Gangulee-W10: 14, M15: 36, S41: 16, S55: 15.

Thuidium philibertii Limpr. -W10: 14, M15: 33, 36, E29: 36, E31: 36.

— recognitum (Hedw.) Lindb. - E29: 36.

Timmia austriaca Hedw. - E29: 36.

_ * comata Lindb. et Arnell - E34: 9.

— *megapolitana Hedw. - M16: 29.

*Timmiella corniculata (Wahlenb.) Broth. - E37: 34.

Tomentypnum nitens (Hedw.) Loeske - N1: 10, W10: 14, M17: 36, E29: 36, E34: 9, 30, E36: 33, E37: 27, 33, S51: 36, S55: 15, S56: 15, 27.

Tortella arctica (Arnell) Crundw. et Nyholm - E29: 36.

- fragilis (Hook. et Wilson) Limpr. -W10: 14, M13: 36, M14: 36, E29: 36, E37: 8 .

— tortuosa (Hedw.) Limpr. - W10: 14, M15: 33, E29: 36, E31: 36.

Tortula cernua (Huebener) Lindb. - C25: 21.

- hoppeana euryphylla (Hedw.) Sander - N1: 10, W10: 14, E37: 4.

- mucronifolia Schwargr. - M17: 8, E29: 36.
- obtusifolia (Schwargr.) Math. - E29: 36.

- systylia (Schimp.) Lindb. - E31: 36.

Trachycystis flagellaris (Sull. et Lesq.) Lindb. - W9: 28, W10: 14, E37: 8.

- ussuriense (Maack et Regel) T. J. Kop. - W10: 14, S55: 15 .

Trematodon ambiguus (Hedw.) Hornsch. - E31: 36, E37: 37.

Ulota crispa (Hedw.) Brid. - W9: 28, W10: 14, W11: 16, M15: 36, S38: 27, S41: 16.

- drummondii (Hook. et Grev.) Brid. -W9: 28, W10: 14, W11: 16, S41: 16, S55: 15.

Warnstorfia exannulata (B.S.G.) Loeske - N1: 10, 33, W10: 14, M15: 36, C25: 21, E29: 36, E33: 36, E34: 9, 30, E37: 33, S41: 16, S51: 36, S55: 15, S56: 15.

— fluitans (Hedw.) Loeske - N1: 33, W7: 25, W11: 16, W12: 27, C25: 21, E28: 36, S44: 22, S55: 15, S56: 15 .

- pseudostraminea (Muell. Hal.) Tuom. et T. J. Kop. -S44: 22, S55: 15.

- sarmentosa (Wahlenb.) Hedenaes - N1: 10, 33, W10: 14, W11: 16, M17: 36, E29: 36, E34: 30, S38: 27, S46: 27, S55: 15, S56: 15.

- tundrae (Arnell) Loeske - N1: 10, E34: 36, S55: 15.

Weissia controversa Hedw. cf. - E31: 36.

Zygodon rupestris (Lindb. ex C. C. Hartm.) Lindb. ex E. Britton - W9: 28, W11: 16, M13: 36, C25: 21, E26: 36 .

\section{EXCLUDED TAXA}

Amblystegium radicale Schimp. - 10 - specimens are absent in herbarium, determination is doubtful.

Anomodon rostratus (Hedw.) Schimp. - 33 - European species, determination is erroneous.

Aulacomnium acuminatum (Lindb. et Arnell) Kindb. - 33 - redetermined by author as $A$. palustre.

Brachythecium rutabulum (Hedw.) B.S.G. - 25, 27, redetermined by Persson, 10 - specimens be absent in herbarium, determination is doubtful.

Bryum veronense De Not. - 33 - redetermined by author in B. argenteum.

Codriophorus acicularis (Hedw.) P. Beauv. - 10, $16-$ redetermined by H. Bednarek-Ochyra in Codriophorus mollis.

Bucklandiella heterosticha (Hedw.) Bednarek-Ochyra et Ochyra - 30, 33, - redetermined by BednarekOchyra and Ignatova in Bucklandiella sudetica.

Dicranum angustum Lindb. - 8, 10, 15, 30, 33, - all studied specimens redetermined.

D. congestum Brid. - all speciemens redetermined as D. fuscescens and other species.

D. hamulosum Mitt. - 15 - redetermined by E. Ignatova in D. fuscescens.

D. viride (Sull.) Lindb. - (Flora i rastitel'nost... 2002) 
- this species is pointed in some geobotanical paper on determination by Cherdanzeva and other bryologist. However all collections were found to be in fact $D$. fragilifolium.

Encalypta brevicollis (B.S.G.) Bruth ex Aongstr. - 33 - redetermined by author in E. rhaptocarpa

Hamatocaulos vernicosus - 30 - redetermined by author in Sanionia uncinata.

Herzogiella striatella (Brid.) Z. Iwats. - 15 - specimens be absent in herbarium, determinations is doubtful.

Hygroamblystegium varium (Hedw.) Moenk. - 33 redetermined by author in Sciurohypnum reflexum

Hygrohypnum alpinum (Lindb.) Loeske - 10 - redetermined by author in Hygrohypnella duriuscula.

Ochyraea molle - 10 - redetermined by author in $\mathrm{Hy}$ grohypnella bestii (Czernyadjeva, 2000).

Hylocomiastrum umbratum (Hedw.) Fliesch. in Broth. - 33 - redetermined by author in $H$. pyrenaicum.

Mnium ambiguum $\mathrm{H}$. Muell. - 16 - redetermined by author in M. lycopodioides.

Palustriella decipiens (De Not.) Ochyra - 33 - redetermined by author in Drepanocladus aduncus.

Plagiomnium undulatum (Hedw.) T. J. Kop. - 27, 25 European species, determination is erroneous.

Pogonatum aloides Hedw. -4 - according to Savicz (1934), specimens was redetermined by Lindberg to Pogonatum capillare and was collected by Redowsky, i.e. not in Kamchatka.

P. nanum (Hedw.) P. Beauv. - 4, 27, - according to Savicz (1934), was collected by Redowsky, i.e. not in Kamchatka.

Polytrichum remotifolium $\mathrm{P}$. Beauv. $=($ P. glabrum $)-4$ - African species, according to Savicz (1934), determination is doubtful.

Rhynchostegium confertum (Dicks.) B.S.G. -33 - redetermined by author.

Schistidium confertum ( Funck) B.S.G. - 20 - redetermined by E. Ignatova.

S. flexipilum (Lindb. ex Broth.) Roth -14 - redetermined by E. Ignatova in $S$. frigidum.

S. strictum (Turner) Mart. - 14 - redetermined by E. Ignatova in S. papillosum.

Sphagnum imbricatum (Hornsch.) Russow - (Jensen, 1909) - according to Savicz (1932), was collected by Redowsky, i.e. not in Kamchatka.

S. subtile (Russ.) Warnst. -30 - specimens is absent in herbaria.

\section{ACKNOWLEDGEMENTS}

I'm very grateful to Dr. M.S. Ignatov and E.A. Ignatova for constant help in determination difficult taxa of mosses. I am very thankful to Dr. O.M. Afonina, Dr. H. Bednarek-Ochyra, Dr. G.Ya. Ukraynskaya, Dr. V.A. Zolotov for determination of the some specimens. I express my sincere thanks to O.A. Chernyagina for the organisation of field investigations and provided some collections of Kamchatka mosses. I am indebted to Dr. V.Yu. Neshataeva, M. Vyatkina for help in field investigations, to Dr. V.A. Bakalin for collection of mosses, and to Prof. W. B. Schofield for correction of English. The researches was supported by the RFBR, grants 0304-49593, 05-04-48705.

\section{SOURCES OF INFORMATION, CITED IN LIST OF MOSSES OF KAMCHATKA}

1 - Afonina \& al., 2004.

2 - Bednarek-Ochyra \& Czernyadjeva, 2003.

3 - Bednarek-Ochyra, 2004.

4 - Bridel-Brideli, 1826, 1827.

5 - Cherdantseva, 1978.

6 - Cherdantseva, 1989.

7 - Cherdantseva, 1993.

8 - Cherdantseva, \& Osipov. 1998.

9 - Cherdantseva, 2003.

10 - Czernyadjeva, 1995a.

11 - Czernyadjeva, $1995 \mathrm{~b}$.

12 - Czernyadjeva, 1995c.

13 - Czernyadjeva, 2000.

14 - Czernyadjeva, 2002a.

15 - Czernyadjeva \& Potemkin, 2002.

16 - Czernyadjeva \& Potemkin, 2003.

17 - Czernyadjeva, 2004.

18 - Czernyadjeva, \& Ignatova, 2004.

19 - Czernyadjeva, 2005a.
20 - Czernyadjeva, 2005b.

21 - Czernyadjeva \& Afonina, 2005.

22 - Czernyadjeva \& al, 2005.

23 - Hooker \& Arnott, 1841.

24 - Ignatova \& Munoz, 2004.

25 - Lazarenko, 1940; 1941a; 1941b; 1945.

26 - Melin, 1924.

27 - Mueller, 1927.

28 - Neshataeva \& al, 2005a.

29 - Persson, 1970.

30 - Rastitel'nost ..., 1994.

31 - Savicz, 1932.

32 - Savicz, 1934.

33 - Vyunova, 1991.

34 - Wahlenberg, 1811.

35 - Wilson, 1858.

36 - Non published data of author.

37 - Non published data of the herbarium LE.

38 - Non published data of E.A. Ignatova (MW).

39 - Blom, 1996. 


\section{Literature Cited}

[AFONINA, O.M., I.V. CZERNYADJEVA, G.Ya. DOROSHINA-UKRAINSKAJA, L.E. KURBATOVA \& E.Yu. KUZMINA] АФОНИНА, О. М., И. В. ЧЕРНЯДЬЕВА, Г. Я. ДОРОШИНА-УКРАИНСКАЯ, Л. Е. КУРБАТОВА., Е. Ю. КУЗЬМИНА. 2004. Эксикаты мохообразных России и сопредельных государств. ЧастьV. - [Bryophyta Rossica et civitatum collimitanearum exsiccata. Fas. V.] СПб. [Sankt-Petersburg]: 28 c.

ARNELL, H.W. 1927. Lebermoose aus Kamtschatka. Hedwigia 67(1/27):110-112.

BEDNAREK-OCHYRA, H. 2004. Codriophorus corrugatus (Briopsida, Grimmiaceae), a new species from East Asia and Southern Alaska. - Bryologist 107(3): 377-384.

BEDNAREK-OCHYRA, H. \& I.V. CZERNYADJEVA. 2003. Racomitrium carinatum Cardot. - J. Bryol. 25: 218-219.

[BLAGODATSKICH, L.S. \& J. DUDA] БЛАГОДАТСКИХ, Л.С., И. ДУДА. 2001. К флоре печеночных мхов Камчатского полуострова. - [On liverwort flora of Kamchatka Peninsula] Новости сист. низи. раст. [Novosti Sist. Nizsh. Rast.] 34: 218-220.

BLOM, H. H. 1996. A revision of Schistidium apocarpum complex in Norway and Sweden. - Bryoph. Bibl. 49: 1-333.

[BOCZ, M.S. \& E.O. KUZMINA]. БОЧ, M.C., Е.О. КУЗЬМИНА. 1991.0 сфагновых мхах полуострова Камчатка. - [On Sphagna of Kamchatka Peninsula] В кн.: Бриология в СССР, ее достижения и перспективы. Ред. О.Т. Демкив. Конф., посвященная 90-летию со дня рожд. А.C. Лазаренко (10-12 сентября 1991 г.) Львов [In: Demkiv, O.T. (ed.) Briologia v SSSR, ee dostizheniya i perspektivy (Proc. Conf., Lvov, 10-12 Sept. 1991). Lvov]: 35-38.

BRIDEL-BRIDELI, S.E. Bryologia universa. 1826, V. I: 746 p.; 1827, V. II: 856 p.

BROTHERUS, V.F. 1931. Mosses (Bryales). Part 3. - Acta horti Botanici Acad. Scient. XLII (2): 141-180.

[BROTHERUS, V.F.] БРОТЕРУC, В.Ф. 1914. Мхи (Andreaealis, Bryales). Часть 1.- [Mosses (Andreaealis, Bryales). Part 1] В кн.: Федченко Б.А. Флора Азиатской Poccuu, Bbin. 4. [In: Fedchenko B.A. Flora Asian Russia, Vyp. 4]: 1-78.

[BROTHERUS, V.F.] БРОТЕРУC, В.Ф. 1918. Мхи (Bryales). Часть 2. - [Mosses (Bryales). Part 2] В кн.: Федченко Б.А. Флора Азиатской России, Bылn. 13. [In: Fedchenko B.A. Flora Asian Russia, Vyp. 4]: 1-182.

[CHERDANTSEVA, V.Ya.] ЧЕРДАНЦЕВА, В.Я. 1978. Материалы к флоре мхов Корякского национального округа. - [To moss flora of Koryakskij District] $B \kappa н$.: Водоросли, грибы и мхи Дальнего Востока. Владивосток, ДВНЦ АН СССР [In: Vodorosli, griby i mhi Dal'nego Vostoka. Vladivostok, DVNC AN SSSR]: 113-123.

[CHERDANTSEVA, V.Ya.] ЧЕРДАНЦЕВА, В.Я. 1989. Редкие и интересные виды мхов Дальнего Востока CCCP- [Rare and interesting moss species of Far East of USSR] Новости сист. низи. pacm. [Novosti Sist. Nizch. Rast] 26: 157-159.

[CHERDANTSEVA, V.Ya.] ЧЕРДАНЦЕВА, В.Я 1993. Мхи - [Mosses] В кн.: Клочкова, Н.Г. (ред.) Редкие виды растений Камчатской области и их охрана. Петропавловск-Камчатский, Дальневосточное книжное издво, Камчатское Отделение [In:Klochkova, N.G. (ed.) Redkie vidy rastenij Kamchatskoj oblasti $i$ ih ohrana. Petropavlovsk-Kamtchatskij, Dal'nevostochnoe kniznoe izd-vo, Kamchatskoe Otdelenie]: 136-151.

[CHERDANTSEVA, V.Ya.] ЧЕРДАНЦЕВА, В.Я. 2003. Материалы к бриофлоре Кроноцкого биосферного заповедника (Дальний Восток, Камчатка) - [On the bryoflora of Kronotsky Biosphere Nature Reserve (Far East, Kamtchatka] В кн.: Растения в муссоном климате. Материаль III межд. конф. «Растения в муссоном климате». Владивосток. [In: Rasteniya v mussonom klimate. Materialy III mezhd. Konf. Vladivostok]:169-173.

[CHERDANTSEVA, V.Ya. \& S.V. OSIPOV] ЧЕРДАНЦЕВА, В.Я., С.В. ОСИПОВ. 1998. К флоре мхов Камчатского полуострова. - [On moss flora of the Kamchatka Peninsula] Бот. Журн. [Bot. Zhurn.] 83(7): 85-92.

[CZERNYADJEVA, I. V.] ЧЕРНЯДЬЕВА, И.В. 1995а. К флоре листостебельных мхов полуострова Камчатка [On the moss flora of Kamchatka Peninsula] Бот. журн. [Bot. Zhurn.] 80(6): 61-74.

CZERNYADJEVA, I. V. 1995b. Pohlia cardotii (Bryaceae, Musci) found in Eurasia (Russia, Kamtchatka Peninsula). - Ann. Bot. Fennici 32: 137-139.

CZERNYADJEVA, I. V. 1995c. Philonotis yezoana Besch. et Card. ex Card. (Bartramiaceae, Musci) new to Russia. Arctoa 4: 15-16.

CZERNYADJEVA, I. V. 2000. First certain record of Hygrohypnum bestii (Ren. \& Bryhn) Holz. ex Broth. for Eurasia (Russian far East, Kamtchatka Peninsula). - Arctoa 9: $105-108$.

[CZERNYADJEVA, I. V.] ЧЕРНЯДЬЕВА, И.В. 2002a. Листостебельные мхи бассейна р. Левый Кихчик (Дальний Восток, Западная Камчатка) - [Mosses of basin of Left Kihchik River (Far East, West Kamtchatka)]. Arctoa 11: 91-100.

[CZERNYADJEVA, I. V.] ЧЕРНЯДЬЕВА, И.В. 2002b. История изучения бриофлоры полуострова Камчатка [The history of study of bryoflora of Kamtchatka Peninsula] $B$ кн.: Проблемь бриологии на рубеже веков. Материаль межд. совещания, посвященного 90-летию со дня рождения Р.Н. Шлякова (20.06.1912) и И.И. Абрамова (14.07.1912), Санкт-Петербург, 4-6 ноября 2002 г. [In: Problems of bryology at the boundary of centries. Proceedings of the international conference devoted to 90-th anniversary of R..N. Schljakov (20.06.1912) and I.I. Abramov (14.07.1912), Saint Petersburg, November 4-6, 2002]: 67-69.

[CZERNYADJEVA, I. V.] ЧЕРНЯДЬЕВА, И.В. $2002 \mathrm{c}$. Новые находки редких и интересных видов листостебельных мхов на полуострове Камчатка- [New records of rare and interesting species of Bryopsida of Kamchatka Peninsula] В кн.: Сохранение биоразнообразия Камчатки и прилегающих морей. Материаль III научной конф. 27-28 ноября 2002 г. ПетропавловскКамчатский [In:Conservation of biodiversity of Kamchatka and coastal waters. Materials of III scientific 
conference, Petropavlovsk-Kamchatsky, November 27-28, 2002]: 103-107.

[CZERNYADJEVA, I. V.] ЧЕРНЯДЬЕВА, И.В. 2003а. Род Hygrohypnum (Amblystegiaceae, Musci) в России - [The genus Hygrohypnum (Amblystegiaceae, Musci) in Russia] Arctoa 12: 25-58.

[CZERNYADJEVA, I. V.] ЧЕРНЯДЬЕВА, И.В. 2003 b. Редкие мхи Камчатки и вопросы их включения в «Красную Книгу Камчатки» - [The rare mosses of Kamchatka and issues of their including to "The Red Book of Kamchatka"] В кн.: Сохранение биоразнообразия Камчатки и прилегающих морей. Материалы IV научной конф. 18-19 ноября 2003 г. ПетропавловскКамчатский [In:Conservation of biodiversity of Kamchatka and coastal waters. Materials of IV scientific conference, Petropavlovsk-Kamchatsky, November 18-19, 2003]: 118-121.

CZERNYADJEVA, I.V. 2004. Anacamptodon kamchaticus (Amblystegiaceae (Musci), a new species of from the Russian Far East. - Arctoa 13: 5-8.

[CZERNYADJEVA, I. V.] ЧЕРНЯДЬЕВА, И.В. 2005a. О находке редкого листостебельного мха Oligotrichum aligerum (Polytrichaceae) на полуострове Камчатка - [On the record of the rare moss Oligotrichum aligerum (Polytrichaceae) on Kamchatka Peninsula] Бот. журн. [Bot. Zhurn.] 90(1): 60-63.

[CZERNYADJEVA, I. V.] ЧЕРНЯДЬЕВА, И.В. 2005 b. Листостебельные мхи бассейна р. Еловки (Центральная Камчатка). - [Mosses of Elovka River basin (Central Kamchatka)] Новости сист. низи. pacm. [Novosti Sist. Nizsh. Rast.] 39: 269-280.

[CZERNYADJEVA, I. V. \& A.D. POTEMKIN] ЧЕРНЯДЬEВА, И.В., А.Д. ПОТЕМКИН 2002. Флора мохообразных - [The bryoflora] В кн.: Флора и растительность южной Камчатки (на примере Южно-Камчатского Федерального заказника) (ред. В.Ю. Нешатаева). Петропавловск-Камчатский[In: V.Ju. Neshataeva (ed.). Flora i rastitel'nost'juzhnoj Kamchatki (na primere JuzhnoKamchatskogo Federal'nogo zakaznika). PetropavlovskKamchatskij]: 44-66.

[CZERNYADJEVA, I. V. \& A.D. POTEMKIN] ЧЕРНЯДЬEBA, И.В., А.Д. ПОТЕМКИН. 2003. К флоре мохообразных юго-западной Камчатки (Российский Дальний Восток) - [ On the bryophyte flora of South-West Kamchatka (Russian Far East)] Arctoa 12: 59-74.

CZERNYADJEVA, I.V. \& E.A. IGNATOVA. 2004. Pohlia tundrae Shaw (Bryaceae, Musci) in Russia. - Arctoa 13: 29-32.

[CZERNYADJEVA, I. V. \& O.M. AFONINA] ЧЕРНЯДЬEВА, И.В., О.М. АФОНИНА. 2005. Мхи окрестностей пос. Пущино (Центральная Камчатка) -[Mosses of vicinity of Pushchino Settlment (Central Kamchatka)] Актуальные проблемы бриологии. Сборник статей по материалам международного совещания, посвященного 90-летию со дня рождения А.Л. Абрамовой. 22-25 ноября, 2005, СПб. [Aktualnye problemy briologii. Proceedings of the international meeting devoted to the 90 th anniversary of A. L. Abramova. 22-25 November 2005, Sankt-Peterburg]: 191-198.
[CZERNYADJEVA, I. V., A.D. POTEMKIN \& V.I. ZOLOTOV] ЧЕРНЯДЬЕВА, И.В., А.Д. ПОТЕМКИН, В.И. ЗОЛОТОВ. 2005. Мохообразные (Bryophyta) окрестностей Мутновских горячих источников (Южная Камчатка) - [Bryophytes of vicinity of Mutnovsky hot springs ( Southern Kamchatka) ] Бот. журн. [Bot. Zhurn.] 90(1): 23-39.

ERMAN, A. 1835. Verzeichniss von Thieren and Pflanzen, welche auf einer Reise um die Erde gesammelt wurden. P. 54 .

[FLORA I RASTITELNOST...2002] ФЛОРА И РАСТИТЕЛЬНОСТЬ Южной Камчатки (на примере ЮжноКамчатского Федерального заказника). 2002. - [Flora and vegetation of Southern Kamchatka (the South-Kamchatka Nature Reserve)] Петропавловск-Камчатский, Камчатский печатный двор [Petropavlovsk-Kamchatsky, Kamchatsky pechatnyj dvor ], 299 c.

HOOKER, W.J. \& G.A.W. ARNOTT. 1841. The Botany of captain Beechey's voyage. London: 119-120.

HULTEN, E. 1927. Flora of Kamtchatka and the Adjacent Islands. 1. - Kungl. Sv. Vetensk. Akad. Handl. Stockholm, Ser. 3. Bd. 5, Hf 1: 1-346.

IGNATOVA, E.A. \& J. MUNOZ .2004. The genus Grimmia Hedw. (Grimmiaceae, Musci) in Russia. - Arctoa 13:101182.

JENSEN, C. 1909. Musci Asiae borealis. Sphagna. - Kgl. Svenska Vet.-Akad. Handl. Upsala.

KABIERSCH, W. 1937 (1938). Studien uber die ostasiatischen Arten einiger Laubmoosfamilien II (Rhizogoniaceae, Bartramiaceae, Aulacomniaceae, Meeseaceae). - Hedwigia 77(2/3): 71-136.

[KHARKEVICH, S.S.] ХАРКЕВИЧ, C.C. 1981. Определитель сосудистых растений Камчатской области. [Hand-book of vascular plants of Kamchatka Region] $M$., Наука [Moskwa, Nauka]: $411 \mathrm{c.}$

[KOMAROV, V.L.] КOMAPOB, В.Л. 1912. Путешествие по Камчатке в 1908-1909 гг. - [Voyage on Kamtchatka in 1908-1909] В кн.: Камчатская экспедиџия Ф.П. Рябушинского. Бот. Отд. Bыл. 1. [Kamchatskaya ekspediciya F.P. Ryabushinskogo. Bot. otd. Vyp. 1]: $456 \mathrm{c}$.

KOPONEN, T. 1981. A sinopsis of Mniaceae (Bryophyta). VI. Southeast Asian taxa. - Acta Bot. Fennica 117: 1-34.

KOPONEN, T. 1994. Cinclidiaceae, Mniaceae and Plagiomniaceae from Minshan Range, northwestern Sichuan, China. - Hikobia 11(4): 387-406.

[LAZARENKO, A. S.] ЛАЗАРЕНКО, А.С. Листяні мохи Радянського Далекого Сходу. - [The mosses of the Soviet Far East] Бот. журн. АН УРСР [Journ. Bot. Acad. Sci. Urss. Ukraine]: 1940 - 1(3-4): 59-100; 1941a - 2(1): 5195; 1941b-2(2): 271-308; 1945 - 2(3): 185-216.

LINDBERG, S.O. \& H.W. ARNELL. 1889-1900. Musci Asiae Borealis - K.V.A. Handl. II Bd. 28 (10): 1-163.

MELIN, E. 1924. Some information to the Sphagnum-flora of Kamtschatka - Bryologist 17: 88-90.

MUELLER, C. 1849-1851. Synopsis muscorum frondosorum omnium hucusque cognitorum. Pars I, II. 
MUELLER, H. 1927. Die Laubmose Kamtschatkas. Hedwigia 67: 86-98.

[NESHATAEVA, V.Yu., I.V. CZERNYADJEVA \& V.Yu. NESHATAYEV]. НЕШАТАЕВА, В.Ю., И.В., ЧЕРНЯДЬEВА, В.Ю. НЕШАТАЕВ. 1997. Растительный покров территории Нижне-Кошелевских термальных источников (Южная Камчатка) - [The plant cover of the Nizhne-Koshelevsk hot springs territory (Southern Kamchatka)] Бот. журн. [Bot. Zhurn.] 82(11): 65-79.

[NESHATAEVA, V.Yu., D.E. GIMEL'BRANT, E.S. KUZNETSOVA \& I.V. CZERNYADJEVA] HEШATAEBA, B.Ю., Д.Е. ГИМЕЛЬБРАНТ, Е.С. КУЗНЕЦОВА, И.В. ЧЕРНЯДЬЕВА. 2003. Ценотические, бриофлористические и лихенобиотические особенности коренных старовозрастных каменноберезовых лесов юго-западной Камчатки. - [The species composition of vascular plants, mosses and lichens and the community structure features of pristine old-growth stone-birch forests of south-west Kamchatka] В кн.: Сохранение биоразнообразия Камчатки и прилегающих морей. Доклады III научной конф. 26-27 ноября 2002 г. Петропавловск-Камчатский [In:Conservation of biodiversity of Kamchatka and coastal waters. Proceedings of III scientific conference, Petropavlovsk-Kamchatsky, November 26-27, 2002]: 100-123.

[NESHATAEVA, V.Yu., O.V. CHERNYAGINA, I.V. CZERNYADJEVA D.E., GIMEL'BRANT, E.S. KUZNETSOVA \& V.E. KIRICHENKO] HЕШATAEBA, B.Ю., O.A. ЧЕРНЯГИНА, И.В. ЧЕРНЯДЬЕВА, Д.Е. ГИМЕЛЬБРАНТ, Е.С. КУЗНЕЦОВА, В.Е. КИРИЧЕНКО 2004. Коренные старовозрастные еловые леса бассейна реки Еловка, Центральная Камчатка (ценотические, бриофлористические и лихенобиотические особенности) [Pristine old-growth spruce forests of the Yelovke River basin (Central Kamchatka): the species composition of vascular plants, mosses and lichens and the community structure features] В кн.: Сохранение биоразнообразия Камчатки и прилегающих морей. Доклады IV научной конф. 18-19 ноября 2003 г. Петропавловск-Камчатский [In:Conservation of biodiversity of Kamchatka and coastal waters. Proceedings of IV scientific conference, Petropavlovsk-Kamchatsky, November 18-19, 2003]: 100-124.

[NESHATAEVA, V.Yu., I.V. CZERNYADJEVA, D.E. GIMEL'BRANT, E.S. KUZNETSOVA, V.Yu. NESHATAEV, O.V. CHERNYAGINA \& M.V. DULIN] HEШAТАЕВА, В.Ю., И.В. ЧЕРНЯДЬЕВА, Д.Е. ГИМЕЛЬБРАНТ, Е.С. КУЗНЕЦОВА, В.Ю. НЕШАТАЕВ, О.А. ЧЕРНЯГИНА, М.В. ДУЛИН. 2005а. Пойменные леса юго-западной Камчатки (флористическая и фитоценотическая характеристика) - [Pristine flood-plain forests of south-west Kamchatka (species composition and the community characteristics)] В кн.: Сохранение биоразнообразия Камчатки и прилегающих морей. Доклады V научной конф. 22-24 ноября 2004 г. ПетропавловскКамчатский [In: Conservation of biodiversity of Kamchatka and coastal waters. Proceedings of V scientific conference, Petropavlovsk-Kamchatsky, November 18-19, 2004]: 70-102.

[NESHATAEVA, V.Yu., O.V. CHERNYAGINA \& I.V. CZERNYADJEVA] HEШATAEBA, B.Ю., O.A. ЧЕРНЯГИНА, И.В. ЧЕРНЯДЬЕВА. 2005b. Редкие растительные сообщества термальных местообитаний района
Мутновского вулкана (Южная Камчатка) - [Rare communities of the hot springs surroundings in the Mutnovsky volcano area (Southern Kamchatka)] Бom. журн. [Bot. Zhurn.] 90(5): 731-748.

NOGUCHI, A. 1989. Illustrated moss flora of Japan. III Hattori Botanical Laboratori: 493-742.

PERSSON, H. 1970. Contribution to the bryoflora of Kamtchatka. - Revue bryologique et lichenologique XXXVII (2): 209-221.

[RASTITEL'NOST ... 1994]. РАСТИТЕЛЬНОСТЬ Кроноцкого государственного заповедника (Восточная Камчатка) 1994. - [Vegetation of Kronotzkij State Reserver (Eastern Kamchatka)]. СПб, БИН РАН. [Sankt-Petersburg, BIN RAN]: 232 pp.

[SAVICZ, L. I.] САВИЧ, Л.И. 1932. Флора торфяных мхов Камчатки. - [Flore des Sphaignes de Kamtchatka] Известия ботанического сада, Ленинград Ан СССР, 1931. [Izvestita botanicheskogo sada, Leningrad, Inst. Bot. Acad., sci. URSS, 1931]: 415-480.

[SAVICZ, L. I.] САВИЧ, Л.И. 1934. К флоре мхов Камчатки. - [Zur Moosflora Kamtschatka (Fam. Funariaceae, Aulacomniaceae, Climaciaceae, Rhytidiaceae, Polytrichaceae)] Cпоровые растения. Тр. БИН АН СССР. Cep. III, bыn. 2. [Sporovye rasteniya. Tr. BIN AN SSSR. Ser. II, Fasc. 2]: 257-296.

[SAVICZ-LYUBITSKAYA, L.I. \& Z.N. SMIRNOVA] САВИЧ-ЛЮБИЦКАЯ, Л.И., З.Н. СМИРНОВА. 1970. Определитель листостебельных мхов СССР. Верхоплодные мхи. - [The Handbook of mosses of the USSR. The mosses acrocarpous] Л., Наука [Leningrad, Nauka] : 824 c.

[VYUNOVA, G. V.] ВьЮНОВА, Г.В. 1991. Материалы к бриофлоре Камчатки (зеленые мхи). - [Contributions to bryoflora of Kamtchatka (mosses)] В кн.: Бриология $в$ СССР, ее достижения и перспективы. Ред. О.Т. Демкив. Конф., посвыщенная 90-летию со дня рожд. А.С. Лазаренко (10-12 сентября 1991 г.) Львов [In: Demkiv, O.T. (ed.) Briologia v SSSR, ee dostizheniya i perspektivy (Proc. Conf., Lvov, 10-12 Sept. 1991). Lvov]: 47-51.

WAHLENBERG, G. 1811. Kamtschadalische Laub- und Lebermoose, gesammelt auf der russischen Entdeckungsreise von dem Herrn Hofrath Tilesius. - Magazin $f$. die neuesten Entd. in der ges. Naturk. 5: 289-297.

WARNSTORF, C. 1911-1917. Zur Bryo-Geographie des Russischen Reiches. - Dresden. 300 p.

WEINMANN, J.A. 1845. Syllabus muscorum frondosorum hucusque in imperio rossico collectorum. - Extr. du Bullet. de la Societe Imp. des Natur. de Moscou. XVIII: 1-149.

WILSON, W. 1858. Musci. - In: Seeman B. Beitrag zur Kryptogamen-Flora Kamtschatkas. Bonplandia Jahrg. VI: 212 .

[YAKUBOV, V.V. \& O.A. CHERNYAGINA] ЯКУБОВ, B.В., О.А. ЧЕРНЯГИНА. 2004. Каталог флоры Камчатки (сосудистые растения). - [Katagol of the flora of Kamchatka (vascular plants)] Петропавловск-Камчатский, «Камчатпресс» [Petropavlovsk-Kamchatsky, "Kamchatpress"], 165 c.

ZOLOTOV, V.I. 2000. The genus Bryum (Bryaceae, Musci) in the Middle European Russia. - Arctoa 9: 155-232. 


\section{SYNOMYMS}

Acrocladium cuspidatum (L.) Lindb. = Calliergonella cuspidata

Amblystegium kochii B. S. G. = Hygroamblystegium humile

Amblystegium riparium (L.) B. S. G. = Leptodictyum riparium

Amblystegium saxatile Schimp. = Amblystegium radicale

Amblystegium varium (Hedw.) Lindb. = Hygroamblystegium varium

Anisothecium squarrosum (Starke) Lindb. = Dicranella palustris

Anisothecium squarrosum var. tenellum Broth. = Dicranella palustris

Anosothecium crispum (Schreb.) Lindb. = Dicranella crispa

Brachythecium collinum (Schleich. ex Muell. Hal.) B. S. G. = Brachytheciastrum collinum

Brachythecium dovrense (Limpr.) Schljakov = Sciurohypnum glaciale var dovrense

Brachythecium glaciale B.S.G. = Sciurohypnum glaciale

Brachythecium latifolium Kindb. = Sciurohypnum latifolium

Brachythecium oedipodium (Mitt.) A. Jaeger = Sciurohypnum oedipodium

Brachythecium plumosum (Hedw.) B.S.G. = Sciurohypnum plumosum

Brachythecium populeum (Hedw.) B.S.G. = Sciurohypnum populeum

Brachythecium reflexum (Starke) Schimp. = Sciurohypnum reflexum

Brachythecium starkei (Brid.) B.S.G. = Sciurohypnum starkei

Brachythecium unicifolium Broth. et Paris = Sciurohypnum unicifolium

Bryoxiphium savatieri (Husn.) Mitt. = B. norvegicum var. japonicum

Bryum cernuum $(\mathrm{Sw}$.) Lindb. $=$ B. uliginosum

Bryum imbricatum (Schwaegr.) B. S. G. = B. amblyodon

Bryum inclinatum (Sw.) B.S.G. = B. amblyodon

Bryum neodamense Itzig. var. ovatum (Jur.) Lindb. et Arnell = B. subneodamense

Calliergon sarmentosum (Wahlenb.) Kindb. = Warnstorfia sarmentosa

Calliergon sarmentosum var. fallaciosum (Milde) Roth $=$ ?Warnstorfia sarmentosa

Calliergon stramineum (Dicks. ex Brid.) Kindb. = Straminergon stramineum

Campthothecium lutescens (Hedw.) B.S.G. = Homalothecium lutescens

Campthothecium trichoides (Neck.) Broth. = Tomentypnum nitens
Campylium chrysophyllum (Brid.) Lange $=$ Campyliadelphus chrysophyllus

Campylium hispidulum (Brid.) Mitt. = Campylidium hispidulum

Campylium polygamum (B.S.G.) C. E. O. Jensen = Drepanocladus polygamus

Campylium sommerfeltii (Myr.) Lange $=$ Campylidi um sommerfeltii

Campylium zemliae C. E. O. Jensen = Drepanocladus arcticus

Campylium adscendens (Lindb.) Perss. $=$ Herzogiella adscendens

Ceratodon purpureus (Hedw.) Brid. var. rotundifolium Berggr. $=$ Ceratodon heterophyllus

Cirriphyllum cirrhosum (Schwaegr.) Grout $=$ Brachyth ecium cirrosum

Cratoneuron commutatum (Hedw.) G. Roth $=$ Palustriella commutata

Cratoneuron decipiens $($ De Not.) Loeske $=$ Palustriella decipiens

Cratoneuron filicinum (L.) Roth. var. fallax (Brid.) Moenkem. $=$ C. filicinum

Cratoneuron glaucum (Lam.) Broth. = Palustriella commutata

Desmatodon cernuus (Huebener) B.S.G. $=$ Tortula cernua

Desmatodon latifolius (Hedw.) Brid. = Tortula hoppeana

Desmatodon systylius Schimp. $=$ Tortula systylia

Dicranoweisia crispula (Hedw.) Lindb. = Hymenoloma crispulum

Dicranoweisia intermedia J. J. Amman. = Hymenoloma intermedia

Dicranum fuscescens Turner var. congestum (Brid.) Husn. $=$ Dicranum fuscescens

Dicranum fuscescens Turner var. flexicaule (Brid.) Wilson $=$ Dicranum flexicaule

Dicranum rupincola (Kindb.) Press. $=$ D. acutifolium

Dicranum undulatum Brid. $=D$. bergeri

Dicranum undulatum Ehrh. $=$ Dicranum polysetum

Ditrichum tenuifolium (Schrad.) Lindb. $=$ D. cylindricum

Drepanocladus badius (Hartm.) Roth = Loeskypnum badium

Drepanocladus exannulatus (B. S. G.) Warnst. $=$ Warnstorfia exannulata

Drepanocladus exannulatus var. tundrae (Arnell) Dietz. $=$ Warnstorfia tundrae

Drepanocladus fluitans $($ L.) Warnst. $=$ Warnstorfia fluitans

Drepanocladus intermedius (Lindb.) Warnst. $=$ Limprichtia cossonii

Drepanocladus lycopodioides (Schwaegr.) Warnst. = Pseudocalliergon lycopodioides 
Drepanocladus pseudostramineus (C. Mull.) G. Roth = Warnstorfia pseudostraminea

Drepanocladus revolvens $(\mathrm{Sw}$.$) Warnst. =$ Limprichtia revolvens

Drepanocladus uncinatus (Hedw.) Warnst. $=$ Sanionia uncinata

Drepanocladus wilsonii (Schimp.) Moenkem. $=D$. sendtneri

Encalypta extinctoria (L.) Sw. = E. vulgaris

Eurhynchium hians (Hedw.) Sande Lac. $=$ Oxyrrhynch ium hians

Eurhynchium pulchellum (Hedw.) Jenn. = Eurhynchiastrum pulchellum

Grimmia alpestris Schleich. var. stomata Loeske f. sessitana (De Not.) Loeske $=G$. reflexidens

Grimmia alpicola $\mathrm{Sw} .=$ Schistidium rivulare

Grimmia sessitana De Not. $=G$. reflexidens

Helodium lanatum (Stroem) Broth. $=H$. blandowii

Helodium sachalinense $($ Lindb.) Broth. $=$ Echinophyllum sachalinensis

Hygrogrimmia mollis (B.S.G.) Loeske = Grimmia mollis

Hygrohypnum bestii (Renauld \& Bryhn) Holz. ex Broth. = Hygrohypnella bestii

Hygrohypnum cochlearifolium (Venturi ex De Not.) Broth. $=$ Ochyraea cochlearifolium

Hygrohypnum duriusculum (De Not.) D. W. Jamieson = Hygrohypnella duriuscula

Hygrohypnum molle $(\mathrm{Hedw}$.) Loeske $=$ Ochyraea molle

Hygrohypnum ochraceum (Turner ex Wilson) Loeske = Hygrohypnella ochraceum

Hylocomium brevirostre (Ehrh.) B. S. G. = Loeskeobryum brevirostre

Hylocomium proliferum (L.) Lindb. $=H$. splendens

Hylocomium pyrenaicum (Spruce) Lindb. $=$ Hylocomiastrum pyrenaicum

Hylocomium umbratum (Hedw.) B. S. G. = Hylocomiastrum umbratum

Hypnum arcuatum Lindb. $=$ Calliergonella lindbergii Hypnum bambergeri Schimp. $=$ Stereodon bambergeri Hypnum callichroum Brid. $=$ Stereodon callichroum Hypnum hamulosum B.S.G. = Stereodon hamulosum Hypnum holmenii Ando $=$ Stereodon holmenii

Hypnum lindbergii Mitt. $=$ Calliergonella lindbergii

Hypnum pallescens $($ Hedw.) P.Beauv. = Stereodon pallescens

Hypnum plicatulum (Lindb.) A. Jaeger $=$ Stereodon plicatulus

Hypnum pratense L. F. Koch ex Spruce = Breidleria pratensis

Hypnum revolutum (Mitt.) Lindb. $=$ Stereodon revolutus

Hypnum reptile Mich. $=$ Stereodon pallescens

Hypnum vaucheri Lesq. $=$ Stereodon vaucher $i$

Isopterygium elegans (Brid.) Lindb. = Pseudotaxiphyllum elegans
Isopterygium pulchellum (Hedw.) Jaeg. et Sauerb. = Isopterygiopsis pulchella

Isothecium viviparum (Neck.) Lindb. $=$ I. alopecurum

Leptodictyum humile (P. Beauv.) Ochyra = Hygroamblystegium humile

Leskeella nervosa (Brid.) Loeske $=$ Pseudoleskeella nervosa

Lescuraea atrovirens Monk. $=$ Leskuraea incurvata

Lesquereuxia secunda (Arnell) Broth. = Leskuraea secunda

Meesia trichodes Spruse $=$ M. uliginosa

Mniobryum albicans $($ Wahlenb.) Limpr. $=$ Pohlia wahlenbergii

Mniobryum wahlenbergii (Web. et Mohr) Jenn. = Pohlia wahlenbergii

Mnium ambiguum $\mathrm{H}$. Muell. = M. lycopodioides

Mnium cinclidioides (Blytt) Huben. = Pseudobryum cinclidioides

Mnium cuspidatum (L.) Neck. = Plagiomnium cuspidatum

Mnium medium B. S. G. = Plagiomnium medium

Mnium nudum Britt. et Wiliams = Rhizomnium nudum

Mnium punctatum (L.) Reich. = Rhizomnium punctatum

Mnium rostratum Schrad. $=$ Plagiomnium rostratum

Mnium rugicum Brid. = Plagiomnium ellipticum

Mnium seligeri Jur. = Plagiomnium elatum

Mnium trichomanes Mitt. $=$ Plagiomnium acutum

Mnium undulatum (L.) Weis. = Plagiomnium undulatum

Nyholmiella obtusifolia (Brid.) Holmen = Orthotrichum obtusifolium

Orthodicranum flagellare $($ Hedw.) Loeske $=$ Dicranum flagellare

Orthodicranum hamulosum (Mitt.) Broth. $=$ Dicranum hamulosum

Orthodicranum montanum (Hedw.) Loeske . = Dicranum montanum

Orthodicranum strictum (Schleich. ex D. Mohr) Culm. = Dicranum tauricum

Philonotis caespitosa Wils. f. filescens Loeske $=P$. caespitosa

Plagiothecium roeseanum B. S. G. = P. cavifolium

Plagiothecium sylvaticum (Brid.) B. S. G. = P. nemorale

Pogonatum capillare $($ Rich.) Brid. $=P$. dentatum

Pogonatum grandifolium (Lindb.) Jaeg. $=$ P. japonicum

Pogonatum polytrichoides $(\mathrm{L}$.) Brockm. $=$ P. nanum

Polytrichum alpinum L. $=$ Polytrichastrum alpinum

Polytrichum alpinum var septentrionale (Roehl.) Lindb.

$=$ Polytrichastrum norvegicum

Polytrichum alpinum var. silvaticum (Menz.) Lindb. = Polytrichastrum alpinum

Polytrichum attenuatum Menz. $=$ Polytrichastrum for mosum 
Polytrichum commune L. var. minus Weis. $=$ P. commune

Polytrichum commune L. var. uliginosum Hueben. $=P$. commune

Polytrichum contortum Menz. = Pogonatum contortum

Polytrichum gracile Menz. = Polytrichastrum longisetum

Polytrichum gracile var. anomalum (Milde) I. Hag. = Polytrichastrum. longisetum

Polytrichum formosum Hedw. = Polytrichastrum formosum

Polytrichum longisetum Sw. ex Brid. = Polytrichastrum longisetum

Polytrichum pilosum (Weis) Neck. $=P$. piliferum

Polytrichum pilosum var. pusillum Moll. $=$ P. piliferum

Polytrichum remotifolium P.B. $=$ P. glabrum

Polytrichum sexangulare Floerke ex Brid. = Polytrichastrum sexangulare

Pseudoleskea incurvata (Hedw.) Loeske = Leskuraea incurvata

Pseudoleskea patens (Lindb.) Kindb. = Leskuraea patens

Pseudoleskea radicosa (Mitt.) Kindb. = Leskuraea radicosa

Pseudoleskea secunda (Arnell) Broth. = Leskuraea secunda

Racomitrium aciculare (Hedw.) Brid. = Codriophorus acicularis

Racomitrium brevisetum Lindb. = Codriophorus brevisetus

Racomitrium canescens (Hedw.) Brid. = Niphotrichum canescens
Racomitrium canescens (Timm.) Brid. var. ericoides (Web.) B. S. G. = Niphotrichum ericoides

Racomitrium carinatum Cardot $=$ Codriophorus carinatus

Racomitrium ericoides (F. Weber ex Brid.) Brid. = Niphotrichum ericoides

Racomitrium fasciculare (Hedw.) Brid. = Codriophorus fascicularis

Racomitrium heterostichum $(\mathrm{Hedw}$.) Brid. $=$ Bucklandiella heterosticha

Racomitrium hypnoides $(\mathrm{L}$.$) Lindb. =R$. lanuginosum

Racomitrium microcarpon $(\mathrm{Hedw}$.) Brid. $=$ Bucklandi ella microcarpa

Racomitrium sudeticum (Funck) B.S.G. $=$ Bucklandi ella sudetica

Racomitrium sudeticum f. kindbergii Frisvoll $=$ Bucklandiella sudetica $\mathrm{f}$. kindbergii

Rhytidiadelphus calvescens (Wils.) Broth. $=$ Rhytidiadelphus subpinnatus

Scleropodium ornellanum (Molendo) Lorentz $=$ Sciurohypnum ornellanum

Sphagnum apiculatum $\mathrm{H}$. Lindb. $=S$. fallax

Stroemia obtusifolia Brid. $=$ Orthotrichum obtusifoli um

Swartzia montana (Sw.) Hag. = Distichium capillaceum

Tetraplodon bryoides (Zoega) Lindb. $=$ T. mnioides

Tetraplodon bryoides var. cavifolius (Schimp.) Moll. = T. mnioides

Tortula ruralis (Hedw.) Lindb. $=$ Syntrichia ruralis

Tortula euryphylla $\mathrm{R}$. H. Zander $=$ T. hoppeana

Trichodon cylindricus (Hedw.) Schimp. = Ditrichum cylindricum 